\title{
A systematic review on the effect of sweeteners on glycemic response and clinically relevant outcomes
}

\author{
Natasha Wiebe ${ }^{1}$, Raj Padwal' ${ }^{1}$, Catherine Field ${ }^{2}$, Seth Marks ${ }^{3}$, Rene Jacobs ${ }^{2}$ and Marcello Tonelli $i^{*^{*}}$
}

\begin{abstract}
Background: The major metabolic complications of obesity and type 2 diabetes may be prevented and managed with dietary modification. The use of sweeteners that provide little or no calories may help to achieve this objective.

Methods: We did a systematic review and network meta-analysis of the comparative effectiveness of sweetener additives using Bayesian techniques. MEDLINE, EMBASE, CENTRAL and CAB Global were searched to January 2011. Randomized trials comparing sweeteners in obese, diabetic, and healthy populations were selected. Outcomes of interest included weight change, energy intake, lipids, glycated hemoglobin, markers of insulin resistance and glycemic response. Evidence-based items potentially indicating risk of bias were assessed.
\end{abstract}

Results: Of 3,666 citations, we identified 53 eligible randomized controlled trials with 1,126 participants. In diabetic participants, fructose reduced 2-hour blood glucose concentrations by $4.81 \mathrm{mmol} / \mathrm{L}(95 \% \mathrm{Cl} 3.29,6.34)$ compared to glucose. Two-hour blood glucose concentration data comparing hypocaloric sweeteners to sucrose or high fructose corn syrup were inconclusive. Based on two $\leq 10$-week trials, we found that non-caloric sweeteners reduced energy intake compared to the sucrose groups by approximately $250-500 \mathrm{kcal} /$ day $(95 \% \mathrm{Cl} 153,806)$. One trial found that participants in the non-caloric sweetener group had a decrease in body mass index compared to an increase in body mass index in the sucrose group $\left(-0.40 \mathrm{vs} 0.50 \mathrm{~kg} / \mathrm{m}^{2}\right.$, and $-1.00 \mathrm{vs} 1.60 \mathrm{~kg} / \mathrm{m}^{2}$, respectively). No randomized controlled trials showed that high fructose corn syrup or fructose increased levels of cholesterol relative to other sweeteners.

Conclusions: Considering the public health importance of obesity and its consequences; the clearly relevant role of diet in the pathogenesis and maintenance of obesity; and the billions of dollars spent on non-caloric sweeteners, little high-quality clinical research has been done. Studies are needed to determine the role of hypocaloric sweeteners in a wider population health strategy to prevent, reduce and manage obesity and its consequences.

\section{Background}

Non-caloric sweeteners have been available commercially since the late 1800s [1] and their use in food products and as table-top sweeteners is increasing perhaps due in part to aggressive marketing promoting their capacity to induce weight loss and weight maintenance [2,3]. In 2007, non-caloric and/or high-intensity sweeteners accounted for $16 \%$ of the US sweetener

\footnotetext{
* Correspondence: mtonelli-admin@med.ualberta.ca

'Department of Medicine, 13-103 Clinical Sciences Building, University of

Alberta, Edmonton, Alberta, T6G 2G3 Canada

Full list of author information is available at the end of the article
}

market (approximately 0.5 billion USD [4]) and projected sales of these products are expected to exceed one billion USD by 2014 [5].

Sugar alcohols can also be used as sweetener additives and provide less calories per gram than saccharides (sugars). However because sugar alcohols cause gastrointestinal symptoms in some individuals due to incomplete absorption in the small intestine, they may be used less frequently than saccharides. A variety of different saccharides is commonly used to sweeten foods, such as sucrose, fructose, glucose, maltose, isomaltulose, and fructooligosaccharide (FOS). FOS has half the calories
() Biomed Central

(c) 2011 Wiebe et al; licensee BioMed Central Ltd. This is an Open Access article distributed under the terms of the Creative Commons Attribution License (http://creativecommons.org/licenses/by/2.0), which permits unrestricted use, distribution, and reproduction in any medium, provided the original work is properly cited. 
per gram than sucrose, fructose, or glucose. Most recently, fructose (a highly commercially used sweetener used in combination with glucose as high fructose corn syrup (HFCS)) has been controversially linked with hypertriglyceridemia [6].

The effects of different sweeteners on clinically relevant outcomes such as weight management, blood glucose and lipids have been incompletely studied. The main metabolic complications of obesity and type 2 diabetes may be prevented and managed in full or in part with dietary modification, including the use of sweeteners that provide little or no calories (hypocaloric sweeteners) [7-10].

This review systematically summarizes the available randomized trial evidence to determine the comparative effectiveness of sweetener additives (non-caloric, sugar alcohols, and saccharides; Table 1) in food.

\section{Methods}

This systematic review was conducted and reported according to guidelines [11].

\section{Data sources and searches}

We did a comprehensive search designed by a MLIStrained librarian to identify all randomized controlled trials (RCTs) comparing sweeteners in generally healthy, overweight/obese and/or diabetic participants. We included only trials published in English as full peerreviewed manuscripts. MEDLINE (1950 to January 13, 2011), EMBASE (1980 to January 13, 2011), CENTRAL (January 13, 2011), and CAB (January 13, 2011) were searched. No existing systematic reviews were found. The specific strategies used are provided in Additional File 1 . The citations and abstracts were screened by two reviewers to identify pertinent trials. Any study considered potentially relevant by one or both reviewers was retrieved for further consideration.

\section{Study selection}

We considered non-caloric sweeteners to include highintensity caloric sweeteners that are functionally noncaloric simply due to extremely low doses (for example, aspartame). Each potentially relevant study was

Table 1 Description of sweeteners

\begin{tabular}{|c|c|c|}
\hline $\begin{array}{l}\text { Sweetener } \\
\text { Commercial products }\end{array}$ & Nutritive, $\mathrm{kcal} / \mathrm{g}$ & $\begin{array}{l}\text { Sweetness intensity, } \\
\text { relative to sucrose }\end{array}$ \\
\hline \multicolumn{3}{|l|}{ Non-caloric } \\
\hline $\begin{array}{l}\text { Acesulfame-K [83-85] } \\
\text { Sunett }^{\mathbb{B}}\end{array}$ & 0 & 200 \\
\hline $\begin{array}{l}\text { Aspartame }[83-86] \\
\text { Equal }^{\circledR}, \text { NutraSweet }^{\circledR}\end{array}$ & 4 & 180 \\
\hline Cyclamate [83-85] & 0 & $30-50$ \\
\hline $\begin{array}{l}\text { Saccharin [83-85] } \\
\text { Sweet'N Low }{ }^{\circledR} \text {, Sugar } \text { Twin }^{\circledR}, \text { Hermesetas }^{\circledR}\end{array}$ & 0 & $300-500$ \\
\hline $\begin{array}{l}\text { Sucralose }[83-85] \\
\text { Splenda }{ }^{\circledR a}\end{array}$ & 0 & 600 \\
\hline \multicolumn{3}{|l|}{ Sugar alcohol } \\
\hline Hydrogenated starch hydrolysate (HSH) [85] & $\leq 3$ & $0.4-0.9$ \\
\hline Lycasin [87] & 2.4 & 0.75 \\
\hline Maltitol [85] & 3 & 0.9 \\
\hline Sorbitol $[85,86]$ & 2.6 & 0.6 \\
\hline \multicolumn{3}{|l|}{ Saccharide } \\
\hline Fructooligosaccharides (FOS) [88] & 2 & $0.3-0.6$ \\
\hline Fructose $[85,86,89]$ & 4 & $1-2$ \\
\hline Glucose $[86,89]$ & $\geq 4$ & $0.5-1$ \\
\hline $\begin{array}{l}\text { High fructose corn syrup (HFCS) }[85,89] \\
\text { Varieties: HFCS 55, HFCS 42, HFCS } 90\end{array}$ & $\geq 4$ & $\sim 1$ \\
\hline Honey [90] & $\geq 4$ & $1-1.5$ \\
\hline $\begin{array}{l}\text { Isomaltulose }[88,91] \\
\text { Palatinose }\end{array}$ & 4 & 0.5 \\
\hline Maltose $[86,89]$ & $\geq 4$ & 0.5 \\
\hline Sucromalt [88] & 4 & 0.7 \\
\hline Sucrose $[86,89]$ & 4 & 1 (reference) \\
\hline Tagatose [83] & 1.5 & 0.9 \\
\hline Trehalose [92] & 4 & 0.45 \\
\hline
\end{tabular}

${ }^{a}$ Splenda also contains maltodextrin and sometimes dextrose which are both nutritive 
independently assessed by two reviewers for inclusion in the review using predetermined eligibility criteria. Disagreements were resolved by consultation with a third party. Trials with healthy, overweight/obese, and/or diabetic adult ( $\geq 16$ years old) participants meeting the following criteria were eligible for inclusion: parallel or crossover RCTs; weight change, energy intake, lipids, glycated hemoglobin (HbA1C), or insulin resistance were reported; had at least two groups comparing different sweeteners (for example, glucose, fructose, sucrose, other saccharides, sugar alcohols, non-caloric sweeteners: aspartame, saccharin, stevioside, sucralose); and where follow-up was at least one week in duration (see the Box in Additional File 1 for study selection summary). RCTs measuring 2-hour blood (serum or plasma) glucose responses in similar populations without the follow-up requirement were also reviewed. All outcomes selected for study (including weight change) are reversible and thus (providing that order was randomly assigned), a cross-over design should be appropriate. Trials with less than ten participants per group were excluded to improve the efficiency of the work without an appreciable loss of power, and with the possible elimination of some small study bias. Trials aimed at evaluating exercise performance or memory enhancement were excluded. Trials with placebo controls were also excluded as we aimed to investigate comparative effectiveness of different sweeteners, as opposed to exploring the implications of avoiding sweeteners altogether.

\section{Data extraction and quality assessment}

A standardized data extraction method was performed by a single reviewer. A second reviewer checked the extracted data for accuracy. The following properties of each trial were recorded in a database: trial characteristics (country, design, sample size, duration of follow-up); participants (age, gender, co-morbidity (obesity, diabetes mellitus - type 1 and 2), baseline body mass index (BMI), diabetic therapy (insulin, oral antihyperglycemic agents, diet, and so on); sweetener characteristics (type, quantity, schedule); diet (that is, daily caloric content by macronutrient/fiber content); and outcomes. Outcomes included weight change (absolute, BMI), energy intake, lipid measures (total cholesterol, triglycerides, high density lipoprotein (HDL), low density lipoprotein (LDL)), HbA1C, insulin resistance (for example, Homeostatic Model Assessment (HOMA) index), and 2-hour blood glucose (with or without meals).

Risk of bias was assessed using items known to be associated with the magnitude of results (that is, method of randomization, double-blinding, description of withdrawals/dropouts, and allocation concealment) [12,13]. Source of funding was also extracted given its potential to introduce bias [14].

\section{Data synthesis and analysis}

Data were analyzed using Stata 11.1 (http://www.stata. com). Missing standard deviations (SDs) were imputed using the maximum value reported in any included study [15]. Missing correlations for change from baseline and for differences between crossover trial periods were assigned a value of 0.63 , the maximum reported value in the included studies. Changes from baseline means were used in place of final means in parallel randomized trials. For weight change, the baseline value prior to the immediate period was used. The mean difference (MD) was used to summarize outcomes. Due to expected diversity between studies, we decided a priori to combine results using a random effects model (Stata command: metan). Additionally we planned to examine the association between certain variables (population, dose, diet, age, gender, and bias criteria) and the effect of specific sweeteners on outcomes, and publication bias with weighted regression [16], however the available comparisons were too sparse to pool trials with outcomes of one week or less. For 2-hour responses, we pooled comparisons by type of sweetener and ordered the matrix tables by expected order of efficacy [17] (that is, non-caloric sweeteners, sugar alcohols, other saccharides, fructose, sucrose, and glucose). Statistical heterogeneity was quantified using the $\tau^{2}$ statistic (between-study variance) [18]. Furthermore, we explored comparative effectiveness of sweeteners on 2-hour responses using network meta-analysis [19] (specifically, Markov chain Monte Carlo [MCMC] methods within a Bayesian framework) in WinBugs (http://www.mrc-bsu.cam.ac.uk/bugs; code was obtained from Ades et al. [20]). Network analysis extends meta-analysis from simply pooling directly compared treatments (direct evidence) to pooling data from studies not directly compared but linked via one or more common comparators (indirect evidence) by assuming consistency of the evidence [19]. Therefore, this technique facilitates the comparison of any two sweeteners not directly compared in any one study. We used noninformative prior distributions: uniform for the betweenstudy variance (range 0 to 20) and Gaussian for the other parameters (mean 0 and variance 10,000). All chains were run for 10,000 iterations after 1,000 burn-in iterations. Convergence of the MCMC algorithm was assessed using autocorrelation plots. By-population results were generated. Inconsistency in the network (disagreement between direct and mixed evidence) was measured using back-calculations [21]. Ninety-five present Bayesian credible intervals are reported.

\section{Results}

\section{Quantity of research available}

The searches identified 3,666 unique records with no trials found outside the main literature searches. After 


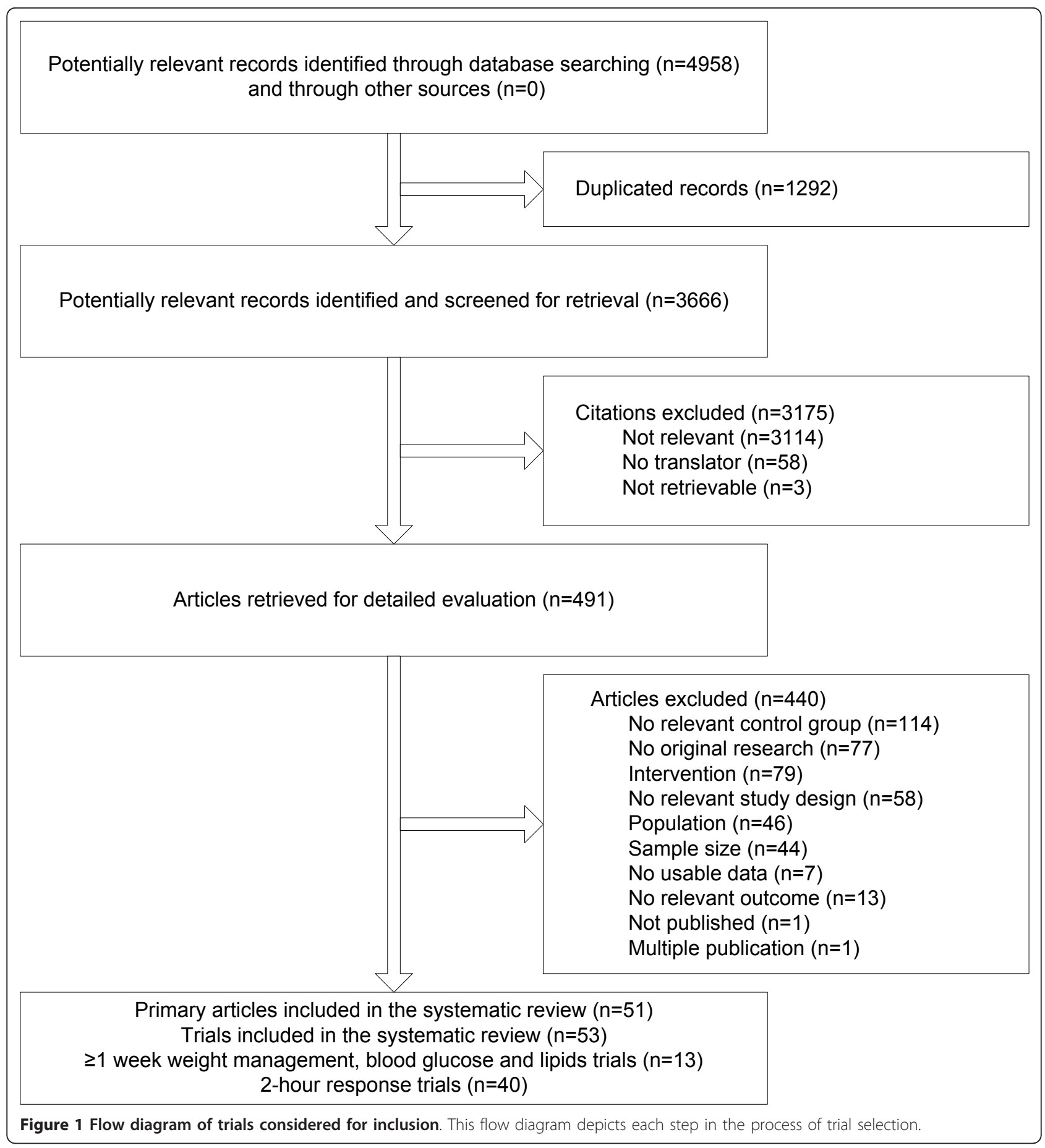

initial screening, 491 articles were retrieved for detailed evaluation (Figure 1) and of these 440 articles were excluded resulting in 53 trials (from 51 publications) that met the selection criteria. Disagreements about the inclusion of studies occurred in $11 \%$ of the articles $($ kappa $=0.71)$. Fourteen were ultimately included. The remaining were excluded for the following reasons: thirteen with no relevant control group, nine with no relevant population, five with no relevant intervention group, four due to study design, four for small sample size, and one for no usable data. The sweeteners studied in eligible trials are described in Table 1.

\section{Characteristics of 2-hour response trials}

Of the forty included trials with 2-hour response data (703 participants; Table 2), three trials compared a non-caloric 
Table 2 Description of included 2-hour response randomized trials

\begin{tabular}{|c|c|c|c|c|c|c|c|}
\hline $\begin{array}{l}\text { Author, } \\
\text { Year, } \\
\text { Country }\end{array}$ & $\begin{array}{c}\text { Population, } \\
\text { Mean BMI }\left(\mathrm{kg} / \mathrm{m}^{2}\right)\end{array}$ & $\begin{array}{c}\text { Sweetener 1: } \\
\text { Type, } \\
\text { Quantity }\end{array}$ & $\begin{array}{l}\text { Sweetener } \\
\text { 2: } \\
\text { Type, } \\
\text { Quantity }\end{array}$ & Medium & $\begin{array}{c}\text { Study } \\
\text { design, } \\
\text { Follow-up, } \\
\text { Sample } \\
\text { size }\end{array}$ & $\begin{array}{c}\text { Mean } \\
\text { age } \\
(y), \\
\% \\
\text { female } \\
\end{array}$ & $\begin{array}{c}\text { Allocation } \\
\text { concealment, } \\
\text { Jadad score, } \\
\text { Funding }\end{array}$ \\
\hline \multicolumn{8}{|c|}{ Non-caloric vs Saccharide } \\
\hline $\begin{array}{c}\text { Gonzalez-Ortiz } \\
{[24]} \\
2009 \\
\text { Mexico }\end{array}$ & $\begin{array}{c}\text { General } \\
23\end{array}$ & $\begin{array}{c}\text { Sucralose (Enterex } \\
\text { Diabetic }{ }^{\mathbb{R}} \text { ) } \\
45 \% \text { once }\end{array}$ & $\begin{array}{l}\text { Mixture } \\
\text { (Glucerna } \\
\text { SR }^{\circledR} \text { ) } \\
50 \% \text { once }\end{array}$ & Drink & $\begin{array}{c}\text { xRCT } \\
21 d \\
\text { periods } \\
\text { (3d wo) } \\
14\end{array}$ & $\begin{array}{l}22 \\
64\end{array}$ & $\begin{array}{l}\text { Unclear } \\
2 \\
-\end{array}$ \\
\hline $\begin{array}{c}\text { Prat-Larquemin } \\
{[22]} \\
2000 \\
\text { France }\end{array}$ & $\begin{array}{l}\text { General } \\
20\end{array}$ & $\begin{array}{l}\text { Aspartame } \\
0.27 \mathrm{~g} \text { once }\end{array}$ & $\begin{array}{l}\text { Sucrose } \\
90 \mathrm{~g} \text { once }\end{array}$ & Cheese & $\begin{array}{c}\text { xRCT } \\
21 \mathrm{~d} \\
\text { periods } \\
\text { (1wk wo) } \\
24\end{array}$ & $\begin{array}{c}23 \\
0\end{array}$ & $\begin{array}{c}\text { Unclear } \\
3 \\
\text { Mixed }^{\text {e }}\end{array}$ \\
\hline $\begin{array}{c}\text { Melchior [23] } \\
1991 \\
\text { France }\end{array}$ & $\begin{array}{c}\text { General } \\
21\end{array}$ & $\begin{array}{l}\text { Aspartame } \\
80 \mathrm{mg} \text { once }\end{array}$ & $\begin{array}{l}\text { Sucrose } \\
50 \mathrm{~g} \text { once }\end{array}$ & Drink & $\begin{array}{c}x R C T \\
21 d \\
\text { periods } \\
10\end{array}$ & $\begin{array}{l}22 \\
70\end{array}$ & $\begin{array}{c}\text { Unclear } \\
1 \\
\text { Public }\end{array}$ \\
\hline \multicolumn{8}{|c|}{ Non-caloric vs Non-caloric } \\
\hline $\begin{array}{c}\text { Horwitz [25] } \\
1988 \\
\text { US }\end{array}$ & $\begin{array}{c}55 \% \text { General/45\% DM2 } \\
<25\end{array}$ & $\begin{array}{l}\text { Aspartame } \\
400 \mathrm{mg} \text { once }\end{array}$ & $\begin{array}{l}\text { Saccharin } \\
135 \mathrm{mg} \\
\text { once }\end{array}$ & $\begin{array}{l}\text { Unsweetened } \\
\text { drink }\end{array}$ & $\begin{array}{c}\text { xRCT } \\
21 \mathrm{~d} \\
\text { periods } \\
\text { (1wk wo) } \\
22\end{array}$ & $\begin{array}{l}41 \\
77\end{array}$ & $\begin{array}{c}\text { Unclear } \\
1 \\
\text { Private }\end{array}$ \\
\hline \multicolumn{8}{|c|}{ Sugar Alcohol vs Saccharide } \\
\hline $\begin{array}{l}\text { Rizkalla [28] } \\
2002 \\
\text { France }\end{array}$ & $\begin{array}{c}50 \% \text { General } \\
25 \\
50 \% \mathrm{DM} 2 \\
27\end{array}$ & $\begin{array}{l}\text { Lycasin } \\
50 \mathrm{~g} \text { once }\end{array}$ & $\begin{array}{l}\text { Glucose } \\
50 \mathrm{~g} \text { once }\end{array}$ & None & $\begin{array}{c}\text { xRCT } \\
21 \mathrm{~d} \\
\text { periods } \\
\text { (1wk wo) } \\
12\end{array}$ & $\begin{array}{c}40 \\
0\end{array}$ & $\begin{array}{l}\text { Unclear } \\
2 \\
\text { Private }\end{array}$ \\
\hline $\begin{array}{l}\text { Nguyen [26] } \\
1993 \\
\text { France }\end{array}$ & $\begin{array}{c}\text { General } \\
\text { - }\end{array}$ & $\begin{array}{l}\text { Lycasin } \\
20 \mathrm{~g} \text { once } \\
\text { Sorbitol } \\
20 \mathrm{~g} \text { once } \\
\text { Xylitol } \\
20 \mathrm{~g} \text { once } \\
\text { Maltitol } \\
20 \mathrm{~g} \text { once }\end{array}$ & $\begin{array}{l}\text { Glucose } \\
20 \mathrm{~g} \text { once }\end{array}$ & None & $\begin{array}{c}\text { xRCT } \\
51 \mathrm{~d} \\
\text { periods } \\
\text { (1wk wo) } \\
10\end{array}$ & $\begin{array}{l}33 \\
50\end{array}$ & $\begin{array}{c}\text { Unclear } \\
1 \\
\text { Private }\end{array}$ \\
\hline $\begin{array}{c}\text { Wheeler [29] } \\
1990 \\
\text { US }\end{array}$ & $\begin{array}{c}33 \% \text { General } \\
24^{4} \\
33 \% \text { DM2 } \\
32 \\
33 \% \text { DM1 } \\
23\end{array}$ & $\begin{array}{l}\text { HSH6075 } \\
50 \mathrm{~g} \text { once } \\
\text { HSH5875 } \\
50 \mathrm{~g} \text { once }\end{array}$ & $\begin{array}{l}\text { Glucose } \\
50 \mathrm{~g} \text { once }\end{array}$ & None & $\begin{array}{c}\text { xRCT } \\
31 \mathrm{~d} \\
\text { periods } \\
18\end{array}$ & $\begin{array}{l}47 \\
50\end{array}$ & $\begin{array}{c}\text { Unclear } \\
2 \\
\text { Mixed }\end{array}$ \\
\hline $\begin{array}{c}\text { Hassinger [27] } \\
1981 \\
\text { Germany }\end{array}$ & $\begin{array}{c}\text { DM1 } \\
-\end{array}$ & $\begin{array}{c}\text { Xylitol } \\
30 \mathrm{~g} \text { once }\end{array}$ & $\begin{array}{l}\text { Sucrose } \\
30 \mathrm{~g} \text { once }\end{array}$ & Meal & $\begin{array}{c}\text { xRCT } \\
21 \mathrm{~d} \\
\text { periods } \\
14\end{array}$ & $\begin{array}{c}29 \\
-\end{array}$ & $\begin{array}{c}\text { Unclear } \\
1 \\
\text { Private }\end{array}$ \\
\hline \multicolumn{8}{|c|}{ Saccharide vs Saccharide } \\
\hline $\begin{array}{c}\text { Maki [30] } \\
2009 \\
\text { US }\end{array}$ & $\begin{array}{l}\text { Obese } \\
35\end{array}$ & $\begin{array}{l}\text { Trehalose } \\
75 \mathrm{~g} \text { once }\end{array}$ & $\begin{array}{l}\text { Trehalose/ } \\
\text { Fructose } \\
75 \mathrm{~g} \text { once } \\
\text { Glucose } \\
75 \mathrm{~g} \text { once }\end{array}$ & None & $\begin{array}{c}\times R C T \\
31 d \\
\text { periods } \\
21\end{array}$ & $\begin{array}{c}50 \\
0\end{array}$ & $\begin{array}{l}\text { Unclear } \\
3 \\
\text { Private }\end{array}$ \\
\hline $\begin{array}{l}\text { Teff [31] } \\
2009 \\
\text { US }\end{array}$ & $\begin{array}{l}\text { Obese } \\
35\end{array}$ & $\begin{array}{c}\text { Fructose } \\
30 \%\end{array}$ & $\begin{array}{c}\text { Glucose } \\
30 \%\end{array}$ & Meals \& Drinks & $\begin{array}{c}\text { xRCT } \\
22 \mathrm{~d} \\
\text { periods } \\
(1 \mathrm{mo} \text { wo) } \\
17\end{array}$ & $\begin{array}{l}33 \\
47\end{array}$ & $\begin{array}{c}\text { Unclear } \\
\quad 1 \\
\text { Public }\end{array}$ \\
\hline $\begin{array}{l}\text { Van Can [52] } \\
2009 \\
\text { Netherlands }\end{array}$ & $\begin{array}{l}\text { Overweight } \\
28\end{array}$ & $\begin{array}{l}\text { Isomaltulose } \\
75 \mathrm{~g} \text { once }\end{array}$ & $\begin{array}{l}\text { Sucrose } \\
75 \mathrm{~g} \text { once }\end{array}$ & None & $\begin{array}{c}\text { xRCT } \\
21 \mathrm{~d} \\
\text { periods } \\
\text { (1wk wo) }\end{array}$ & $\begin{array}{l}31 \\
20\end{array}$ & $\begin{array}{c}\text { Unclear } \\
1 \\
\text { Private }\end{array}$ \\
\hline
\end{tabular}


Table 2 Description of included 2-hour response randomized trials (Continued)

\begin{tabular}{|c|c|c|c|c|c|c|c|}
\hline $\begin{array}{c}\text { Grysman [58] A } \\
2008 \\
\text { Canada }\end{array}$ & $\begin{array}{l}\text { General } \\
24\end{array}$ & $\begin{array}{l}\text { Sucromalt } \\
50 \mathrm{~g} \text { once }\end{array}$ & $\begin{array}{l}\text { HFCS42 } \\
50 \mathrm{~g} \text { once }\end{array}$ & None & $\begin{array}{c}x R C T \\
21 \mathrm{~d} \\
\text { periods } \\
10\end{array}$ & $\begin{array}{l}28 \\
30\end{array}$ & $\begin{array}{c}\text { Unclear } \\
2 \\
\text { Private }\end{array}$ \\
\hline $\begin{array}{c}\text { Grysman [58] B } \\
2008 \\
\text { Canada }\end{array}$ & $\begin{array}{c}\text { General } \\
24\end{array}$ & $\begin{array}{l}\text { Sucromalt } \\
80 \mathrm{~g} \text { once }\end{array}$ & $\begin{array}{l}\text { HFCS42 } \\
80 \mathrm{~g} \text { once }\end{array}$ & None & $\begin{array}{c}x R C T \\
21 d \\
\text { periods } \\
10\end{array}$ & $\begin{array}{l}25 \\
50\end{array}$ & $\begin{array}{c}\text { Unclear } \\
2 \\
\text { Private }\end{array}$ \\
\hline $\begin{array}{c}\text { Grysman [58] C } \\
2008 \\
\text { Canada }\end{array}$ & $\begin{array}{l}\text { General } \\
24\end{array}$ & $\begin{array}{l}\text { Sucromalt } \\
50 \mathrm{~g} \text { once }\end{array}$ & $\begin{array}{c}\text { HFCS42 } \\
50 \mathrm{~g} \text { once } \\
\text { Glucose } \\
50 \mathrm{~g} \text { once }\end{array}$ & Meal & $\begin{array}{c}x R C T \\
31 d \\
\text { periods } \\
20\end{array}$ & $\begin{array}{l}37 \\
60\end{array}$ & $\begin{array}{c}\text { Unclear } \\
2 \\
\text { Private }\end{array}$ \\
\hline $\begin{array}{c}\text { Munstedt [59] } \\
2008 \\
\text { Germany }\end{array}$ & $\begin{array}{c}\text { General } \\
23\end{array}$ & $\begin{array}{l}\text { Glucose/Fructose }{ }^{a} \\
155 \mathrm{~g} \text { once }\end{array}$ & $\begin{array}{c}\text { Honey } \\
221 \mathrm{~g} \text { once }\end{array}$ & None & $\begin{array}{c}\text { xRCT } \\
21 \mathrm{~d} \\
\text { periods } \\
(1 \mathrm{wk} \text { wo }) \\
10\end{array}$ & $\begin{array}{c}28 \\
0\end{array}$ & $\begin{array}{c}\text { Unclear } \\
2 \\
-\end{array}$ \\
\hline $\begin{array}{c}\text { Stanhope [54] } \\
2008 \\
\text { US }\end{array}$ & $\begin{array}{l}\text { General } \\
25\end{array}$ & $\begin{array}{l}\text { HFCS55 } \\
25 \% \text { thrice }\end{array}$ & $\begin{array}{l}\text { Sucrose } \\
25 \% \text { thrice }\end{array}$ & 3 Meals & $\begin{array}{c}\text { xRCT } \\
21 \mathrm{~d} \\
\text { periods } \\
(1 \mathrm{mo} \text { wo }) \\
34\end{array}$ & $\begin{array}{l}35 \\
47\end{array}$ & $\begin{array}{c}\text { Unclear } \\
1 \\
\text { Mixed }\end{array}$ \\
\hline $\begin{array}{c}\text { Bowen [32] } \\
2007 \\
\text { Australia }\end{array}$ & $\begin{array}{l}\text { Overweight/ } \\
\text { Obese } \\
33\end{array}$ & $\begin{array}{l}\text { Fructose } \\
50 \mathrm{~g} \text { once }\end{array}$ & $\begin{array}{l}\text { Glucose } \\
50 \mathrm{~g} \text { once }\end{array}$ & Drink & $\begin{array}{c}\text { xRCT } \\
21 \mathrm{~d} \\
\text { periods } \\
\text { (7d wo) } \\
28\end{array}$ & $\begin{array}{c}57 \\
0\end{array}$ & $\begin{array}{c}\text { Unclear } \\
4 \\
\text { Mixed }\end{array}$ \\
\hline $\begin{array}{c}\text { Chong [33] } \\
2007 \\
\text { UK }\end{array}$ & $\begin{array}{l}\text { General } \\
25\end{array}$ & $\begin{array}{c}\text { Fructose } \\
0.75 \mathrm{~g} / \mathrm{kg} \text { once }\end{array}$ & $\begin{array}{l}\text { Glucose } \\
0.75 \mathrm{~g} / \mathrm{kg} \\
\text { once }\end{array}$ & Drink & $\begin{array}{c}x R C T \\
21 d \\
\text { periods } \\
\text { (6wk wo) } \\
14\end{array}$ & $\begin{array}{l}43 \\
43\end{array}$ & $\begin{array}{c}\text { Unclear } \\
1 \\
\text { Mixed }\end{array}$ \\
\hline $\begin{array}{c}\text { Melanson [55] } \\
2007 \\
\text { US }\end{array}$ & $\begin{array}{l}\text { General } \\
22\end{array}$ & $\begin{array}{l}\text { HFCS55 } \\
30 \% \text { TID }\end{array}$ & $\begin{array}{l}\text { Sucrose } \\
30 \% \text { TID }\end{array}$ & 3 Meals & $\begin{array}{c}x R C T \\
22 d \\
\text { periods } \\
(6 w k \text { wo }) \\
30\end{array}$ & $\begin{array}{c}33 \\
100\end{array}$ & $\begin{array}{c}\text { Unclear } \\
2 \\
\text { Private }\end{array}$ \\
\hline $\begin{array}{c}\text { Visvanathan [34] } \\
2005 \\
\text { Australia }\end{array}$ & $\begin{array}{l}\text { General } \\
26\end{array}$ & $\begin{array}{l}\text { Sucrose } \\
50 \mathrm{~g} \text { once } \\
\text { Fructose } \\
50 \mathrm{~g} \text { once }\end{array}$ & $\begin{array}{l}\text { Glucose } \\
50 \mathrm{~g} \text { once }\end{array}$ & None & $\begin{array}{c}\text { xRCT } \\
31 \mathrm{~d} \\
\text { periods } \\
(3 \mathrm{~d} \text { wo) } \\
10\end{array}$ & $\begin{array}{l}72 \\
60\end{array}$ & $\begin{array}{c}\text { Unclear } \\
1 \\
\text { Public }\end{array}$ \\
\hline $\begin{array}{c}\text { Teff [35] } \\
2004 \\
\text { US }\end{array}$ & $\begin{array}{l}\text { General } \\
23\end{array}$ & $\begin{array}{l}\text { Fructose } \\
30 \% \text { TID }\end{array}$ & $\begin{array}{l}\text { Glucose } \\
30 \% \text { TID }\end{array}$ & 3 Meals & $\begin{array}{c}\text { xRCT } \\
22 \mathrm{~d} \\
\text { periods } \\
(1 \mathrm{mo} \text { wo }) \\
12\end{array}$ & $\begin{array}{c}25 \\
100\end{array}$ & $\begin{array}{c}\text { Unclear } \\
1 \\
\text { Mixed }\end{array}$ \\
\hline $\begin{array}{l}\text { Qin [53] } \\
2003 \\
\text { Japan }\end{array}$ & $\begin{array}{l}\text { General } \\
23\end{array}$ & $\begin{array}{c}\text { Maltose } \\
75 \mathrm{~g} \text { QIDx4h }\end{array}$ & $\begin{array}{l}\text { Sucrose } \\
75 \mathrm{~g} \\
\text { QIDx4h }\end{array}$ & None & $\begin{array}{c}\text { xRCT } \\
21 \mathrm{~d} \\
\text { periods } \\
(1 \mathrm{wk} \text { wo }) \\
10\end{array}$ & $\begin{array}{c}22 \\
0\end{array}$ & $\begin{array}{c}\text { Unclear } \\
1 \\
-\end{array}$ \\
\hline $\begin{array}{l}\text { Vozzo [36] } \\
2002 \\
\text { Australia }\end{array}$ & $\begin{array}{c}50 \% \text { IGT/50\% DM2 } \\
31\end{array}$ & $\begin{array}{l}\text { Fructose } \\
75 \mathrm{~g} \text { once }\end{array}$ & $\begin{array}{l}\text { Glucose } \\
75 \mathrm{~g} \text { once }\end{array}$ & None & $\begin{array}{c}x R C T \\
21 d \\
\text { periods } \\
\text { (5d wo) } \\
20\end{array}$ & $\begin{array}{l}56 \\
40\end{array}$ & $\begin{array}{c}\text { Unclear } \\
1 \\
\text { Mixed }\end{array}$ \\
\hline $\begin{array}{c}\text { Spiller [37] } \\
1998 \\
\text { US }\end{array}$ & $\begin{array}{c}\text { General } \\
\text { - }\end{array}$ & $\begin{array}{l}\text { Sucrose } \\
90 \mathrm{~g} \text { once }\end{array}$ & $\begin{array}{l}\text { Glucose } \\
90 \mathrm{~g} \text { once }\end{array}$ & None & $\begin{array}{c}\text { xRCT } \\
21 \mathrm{~d} \\
\text { periods } \\
\text { (1wk wo) } \\
10\end{array}$ & $\begin{array}{l}29 \\
50\end{array}$ & $\begin{array}{c}\text { Unclear } \\
1 \\
\text { Private }\end{array}$ \\
\hline $\begin{array}{c}\text { Stewart [38] } \\
1997 \\
\text { Canada }\end{array}$ & $\begin{array}{l}\text { General } \\
20-27\end{array}$ & $\begin{array}{l}\text { Fructose } \\
30 \mathrm{~g} \mathrm{SID}\end{array}$ & $\begin{array}{l}\text { Glucose } \\
33.5 \mathrm{~g} \mathrm{SID}\end{array}$ & Meal & $\begin{array}{c}\text { xRCT } \\
21 \mathrm{~d} \\
\text { periods } \\
13\end{array}$ & $\begin{array}{c}25 \\
0\end{array}$ & $\begin{array}{c}\text { Unclear } \\
1 \\
\text { Private }\end{array}$ \\
\hline
\end{tabular}


Table 2 Description of included 2-hour response randomized trials (Continued)

\begin{tabular}{|c|c|c|c|c|c|c|c|}
\hline $\begin{array}{c}\text { Blaak [39] } \\
1996 \\
\text { Netherlands }\end{array}$ & $\begin{array}{c}\text { General } \\
-\end{array}$ & $\begin{array}{l}\text { Sucrose } \\
75 \mathrm{~g} \text { once } \\
\text { Fructose } \\
75 \mathrm{~g} \text { once }\end{array}$ & $\begin{array}{l}\text { Glucose } \\
75 \mathrm{~g} \text { once }\end{array}$ & None & $\begin{array}{c}\text { xRCT } \\
31 \mathrm{~d} \\
\text { periods } \\
(1 \mathrm{wk} \text { wo }) \\
10\end{array}$ & $\begin{array}{c}28 \\
0\end{array}$ & $\begin{array}{l}\text { Unclear } \\
1 \\
\text { Mixed }\end{array}$ \\
\hline $\begin{array}{c}\text { Fukagawa [40] } \\
1995 \\
\text { US }\end{array}$ & General & $\begin{array}{l}\text { Fructose } \\
75 \text { g once }^{d}\end{array}$ & $\begin{array}{l}\text { Glucose } \\
75 \mathrm{~g} \text { once }\end{array}$ & None & $\begin{array}{l}\text { xRCT } \\
21 \mathrm{~d} \\
\text { periods } \\
16\end{array}$ & $\begin{array}{l}47 \\
38\end{array}$ & $\begin{array}{c}\text { Unclear } \\
1 \\
\text { Public }\end{array}$ \\
\hline $\begin{array}{l}\text { Schwarz [41] } \\
1992 \\
\text { Switzerland }\end{array}$ & $\begin{array}{c}43 \% \text { General } \\
21 \\
57 \% \text { Overweight } \\
30\end{array}$ & $\begin{array}{l}\text { Fructose } \\
75 \mathrm{~g} \text { once }\end{array}$ & $\begin{array}{l}\text { Glucose } \\
75 \mathrm{~g} \text { once }\end{array}$ & Meal & $\begin{array}{c}x R C T \\
21 d \\
\text { periods } \\
(4 d \text { wo) } \\
23\end{array}$ & $\begin{array}{c}25 \\
100\end{array}$ & $\begin{array}{l}\text { Unclear } \\
1 \\
\text { Mixed }\end{array}$ \\
\hline $\begin{array}{c}\text { Bukar [42] } \\
1990 \\
\text { US }\end{array}$ & $\begin{array}{c}\mathrm{DM} 2 \\
-\end{array}$ & $\begin{array}{c}\text { HFCS } \\
27 \mathrm{~g}(12.2 \mathrm{~g} \text { Fructose/ } \\
14.8 \mathrm{~g} \text { Glucose) once } \\
\text { Sucrose } \\
33.5 \mathrm{~g} \text { once }\end{array}$ & $\begin{array}{l}\text { Glucose } \\
50 \mathrm{~g} \text { once }\end{array}$ & $\begin{array}{l}\text { HFCS: Tofu frozen } \\
\text { dessert } \\
\text { Sucrose: Ice cream } \\
\text { Glucose: None }\end{array}$ & $\begin{array}{c}\text { xRCT } \\
31 \mathrm{~d} \\
\text { periods } \\
(2 \mathrm{~d} \text { wo }) \\
12\end{array}$ & $\begin{array}{l}51 \\
50\end{array}$ & $\begin{array}{l}\text { Unclear } \\
1 \\
-\end{array}$ \\
\hline $\begin{array}{c}\text { Georgakopoulos } \\
{[43]} \\
1990 \\
\text { Greece }\end{array}$ & General & $\begin{array}{l}\text { Sucrose } \\
20 \mathrm{~g} \text { once }\end{array}$ & $\begin{array}{l}\text { Glucose } \\
20 \mathrm{~g} \text { once }\end{array}$ & None & $\begin{array}{c}\text { xRCT } \\
21 d \\
\text { periods } \\
\text { (3d wo) } \\
17\end{array}$ & $\begin{array}{l}\text { Range } \\
25-40 \\
29\end{array}$ & $\begin{array}{l}\text { Unclear } \\
1 \\
-\end{array}$ \\
\hline $\begin{array}{l}\text { Kawai [56] } \\
1989 \\
\text { Japan }\end{array}$ & $\begin{array}{c}50 \% \text { General } \\
20 \\
50 \% \text { DM2 } \\
23\end{array}$ & $\begin{array}{l}\text { Isomaltulose } \\
50 \mathrm{~g} \text { once }\end{array}$ & $\begin{array}{l}\text { Sucrose } \\
50 \mathrm{~g} \text { once }\end{array}$ & None & $\begin{array}{c}\text { xRCT } \\
21 d \\
\text { periods } \\
(2 d \text { wo }) \\
20\end{array}$ & $\begin{array}{l}39 \\
30\end{array}$ & $\begin{array}{l}\text { Unclear } \\
1 \\
\text { Mixed }\end{array}$ \\
\hline $\begin{array}{l}\text { Schwarz [44] } \\
1989 \\
\text { Switzerland }\end{array}$ & $\begin{array}{c}\text { General } \\
21\end{array}$ & $\begin{array}{l}\text { Fructose } \\
75 \mathrm{~g} \text { once }\end{array}$ & $\begin{array}{l}\text { Glucose } \\
75 \mathrm{~g} \text { once }\end{array}$ & Drink & $\begin{array}{c}\times R C T \\
21 d \\
\text { periods } \\
(4 d \text { wo }) \\
20\end{array}$ & $\begin{array}{l}23 \\
50\end{array}$ & $\begin{array}{l}\text { Unclear } \\
1 \\
\text { Private }\end{array}$ \\
\hline $\begin{array}{c}\text { Simonson [45] } \\
1988 \\
\text { Switzerland }\end{array}$ & DM2/General/Obese ${ }^{f}$ & $\begin{array}{l}\text { Fructose } \\
75 \mathrm{~g} \text { once }\end{array}$ & $\begin{array}{l}\text { Glucose } \\
75 \mathrm{~g} \text { once }\end{array}$ & None & $\begin{array}{c}x R C T \\
21 d \\
\text { periods } \\
\text { (1wk wo) } \\
37\end{array}$ & $\begin{array}{l}53 \\
51\end{array}$ & $\begin{array}{c}\text { Unclear } \\
1 \\
\text { Mixed }\end{array}$ \\
\hline $\begin{array}{l}\text { Jansen [46] } \\
\quad 1987 \\
\text { Netherlands }\end{array}$ & General & $\begin{array}{l}\text { Fructose } \\
75 \mathrm{~g} \text { once }\end{array}$ & $\begin{array}{l}\text { Glucose } \\
75 \text { g once }\end{array}$ & None & $\begin{array}{c}\text { xRCT } \\
21 \mathrm{~d} \\
\text { periods } \\
\text { (1wk wo) } \\
20\end{array}$ & $\begin{array}{l}52 \\
50\end{array}$ & $\begin{array}{l}\text { Unclear } \\
1 \\
\text { Public }\end{array}$ \\
\hline $\begin{array}{l}\text { Tappy [47] } \\
1986 \\
\text { Switzerland }\end{array}$ & General & $\begin{array}{l}\text { Fructose } \\
75 \mathrm{~g} \text { once }\end{array}$ & $\begin{array}{l}\text { Glucose } \\
75 \mathrm{~g} \text { once }\end{array}$ & None & $\begin{array}{c}x R C T \\
21 d \\
\text { periods } \\
(2 d \text { wo }) \\
10\end{array}$ & $\begin{array}{l}27^{c} \\
65^{c}\end{array}$ & $\begin{array}{c}\text { Unclear } \\
1 \\
\text { Mixed }\end{array}$ \\
\hline $\begin{array}{l}\text { Erkelens [57] } \\
1985 \\
\text { Netherlands }\end{array}$ & $\begin{array}{c}\text { 33\% Generalf } / 33 \% \text { DM2/17\% } \\
\text { DM1/17\% insulin infusion DM1 }\end{array}$ & $\begin{array}{c}\text { Honey } \\
\text { (22\% Glucose/26\% } \\
\text { Fructose) SID }\end{array}$ & $\begin{array}{l}\text { Sucrose } \\
49 \% \text { SID }\end{array}$ & $\begin{array}{c}\text { White bread \& } \\
\text { Cheese }\end{array}$ & $\begin{array}{c}x R C T \\
21 d \\
\text { periods } \\
\text { (2d wo) } \\
24\end{array}$ & $\begin{array}{l}47 \\
46\end{array}$ & $\begin{array}{c}\text { Unclear } \\
1 \\
\text { Mixed }\end{array}$ \\
\hline $\begin{array}{c}\text { Samanta [48] } \\
1985 \\
\text { UK }\end{array}$ & $\begin{array}{c}\text { 46\% General/31\% DM1/23\% } \\
\text { DM2 } \\
-\end{array}$ & $\begin{array}{l}\text { Honey } \\
26 \text { g once } \\
\text { Sucrose } \\
26 \text { g once }\end{array}$ & $\begin{array}{l}\text { Glucose } \\
26 \mathrm{~g} \text { once }\end{array}$ & None & $\begin{array}{l}\times R C T \\
31 d \\
\text { periods } \\
26\end{array}$ & $\begin{array}{c}40 \\
-\end{array}$ & $\begin{array}{l}\text { Unclear } \\
1 \\
-\end{array}$ \\
\hline $\begin{array}{c}\text { Bantle [49] } \\
1983 \\
\text { US }\end{array}$ & $\begin{array}{c}\text { 31\% General/38\% DM1/31\% } \\
\text { DM2 } \\
-\end{array}$ & $\begin{array}{l}\text { Sucrose } \\
42 \mathrm{~g} \text { once } \\
\text { Fructose } \\
42 \mathrm{~g} \text { once }\end{array}$ & $\begin{array}{l}\text { Glucose } \\
42 \mathrm{~g} \text { once }\end{array}$ & Meal & $\begin{array}{l}x R C T \\
31 d \\
\text { periods } \\
32\end{array}$ & $\begin{array}{l}41 \\
56\end{array}$ & $\begin{array}{l}\text { Unclear } \\
2 \\
\text { Mixed }\end{array}$ \\
\hline
\end{tabular}


Table 2 Description of included 2-hour response randomized trials (Continued)

\begin{tabular}{|c|c|c|c|c|c|c|c|}
\hline $\begin{array}{c}\text { Crapo [50] } \\
1982 \\
\text { US }\end{array}$ & $\begin{array}{c}\text { 38\% General } / 23 \% \mathrm{IGT}^{\mathrm{f}} / 38 \% \\
\mathrm{DM} 2 \\
-\end{array}$ & $\begin{array}{l}\text { Sucrose } \\
63 \mathrm{~g} \text { once } \\
\text { Sucrose } \\
52 \mathrm{~g} \text { once } \\
\text { Fructose } \\
63 \mathrm{~g} \text { once } \\
\text { Fructose } \\
52 \mathrm{~g} \text { once }\end{array}$ & $\begin{array}{c}\text { Glucose } \\
69.9 \mathrm{~g} \text { once }\end{array}$ & $\begin{array}{c}\text { Sucrose \& } \\
\text { Fructose } 63 \text { g: } \\
\text { cake } \\
\text { Sucrose \& } \\
\text { Fructose } 52 \text { g: ice } \\
\text { cream } \\
\text { None }\end{array}$ & $\begin{array}{c}\times \mathrm{RCT} \\
5 \mathrm{ld} \\
\text { periods } \\
\text { (1d wo) } \\
26\end{array}$ & $\begin{array}{l}43 \\
42\end{array}$ & $\begin{array}{c}\text { Unclear } \\
1 \\
\text { Mixed }\end{array}$ \\
\hline $\begin{array}{c}\text { Mann [51] } \\
1971 \\
\text { South Africa }\end{array}$ & $\begin{array}{c}\text { General } \\
\text { - }\end{array}$ & $\begin{array}{l}\text { Sucrose } \\
60 \mathrm{~g} \text { once }\end{array}$ & $\begin{array}{l}\text { Glucose } \\
60 \mathrm{~g} \text { once }\end{array}$ & Meal & $\begin{array}{c}\text { xRCT } \\
2 \text { 1d } \\
\text { periods } \\
\text { (2d wo) } \\
19\end{array}$ & $\begin{array}{c}\text { Range } \\
20-58 \\
0\end{array}$ & $\begin{array}{c}\text { Unclear } \\
1 \\
\text { Public }\end{array}$ \\
\hline
\end{tabular}

DM1, type 1 diabetes mellitus; DM2, type 2 diabetes mellitus; IGT, impaired glucose tolerance; UK, United Kingdom; US, United States; HFCS, high fructose corn syrup; FOS, fructooligosaccharide; SID, once a day; BID, twice a day; TID, three times a day; QID, four times a day; carb, carbohydrate; xRCT, randomized crossover trial; RCT, parallel randomized controlled trial; wo, washout; max, maximum "-" means the value was not reported in the study, and not described

a'comparable Honey'

${ }^{b}$ contains isomaltulose, fructose, Sucromalt ${ }^{\circledR}$ (sucrose, maltose), and FOS

capproximate because it includes 7 people from other studies written up in the same article

dfactorial trial including $300 \mathrm{mg}$ caffeine or $300 \mathrm{mg}$ vitamin C

eboth public and private sources of funding

f group of participants dropped due to low group sample size

sweetener (aspartame [22,23], or sucralose [24]) to a saccharide (fructose [24] or sucrose [22,23]); one trial compared a non-caloric to another non-caloric (aspartame versus saccharin [25]); four trials compared a sugar alcohol or a malt containing a sugar alcohol (sorbitol [26], xylitol $[26,27]$, maltitol [26], Lycasin [26,28], or a hydrogenated starch hydrolysate (HSH) [29]) to a saccharide (glucose $[26,28,29]$ or sucrose [27]); and thirty-two trials compared a saccharide to another saccharide (glucose [30-51], fructose [31-34,36,38-41,44-47,49,50], mixtures of glucose and fructose [that is, sucrose [34,37,39,42,43,48-57], HFCS $[42,54,55,58]$, honey $[48,57,59]$, glucose/fructose equivalent honey [59]], isomaltulose [52,56], maltose [53], sucromalt [58], trehalose [30], or a mixture of trehalose and fructose [30]). Approximately half of the doses for saccharides were less than the $60 \mathrm{~g} /$ day recommended for diabetic patients on a $2,000 \mathrm{kcal}$ diet; the remainder exceeded $60 \mathrm{~g} /$ day (typically $75 \mathrm{~g}$ ). All of the doses for sugar alcohols exceeded the $10 \mathrm{~g} /$ day recommendation (range 20 to 50 g), which is aimed at limiting gastrointestinal symptoms. None of the four non-caloric sweetener groups were above Acceptable Daily Intake (ADI) values.

Twelve trials included diabetic populations (range mean BMI 23 to $32 \mathrm{~kg} / \mathrm{m}^{2}$ ) [25,27-29,36,42,45,48$50,56,57]$, five trials exclusively studied overweight or obese individuals (range mean BMI 28 to $35 \mathrm{~kg} / \mathrm{m}^{2}$ ) $[30,31,41,45,52]$, and thirty-five trials included generally healthy individuals (range mean BMI 20 to $26 \mathrm{~kg} / \mathrm{m}^{2}$ ). Median mean age was 35 years (range 22 to 72 years) and median sex distribution was $47 \%$ women.

Sample size ranged from 10 to 37 (median 17), three studies $(8 \%)$ had sample sizes $\geq 30$ per group and all were randomized crossover trials. The median Jadad score was 1 (range 1 to 4); no studies reported concealing treatment allocation.

\section{2-hour blood glucose response}

Table 3 reports the results of the direct meta-analysis for all populations in the lower triangle and the mixed evidence from the Bayesian network (Figure 2) in the upper triangle. The network included 36 trials and 610 participants. The direct evidence from all nine comparisons was consistent with the mixed evidence from the network. There was large heterogeneity between trials $\left(\mathrm{I}^{2}{ }_{\mathrm{s}} \mathrm{z} 77 \%\right)$ for three of seven multi-study direct evidence comparisons. Two of the heterogeneous comparisons included a variety of sweeteners (that is, multiple sugar alcohols $\left(\tau^{2}=9.05(95 \%\right.$ CI $\left.2.94,32)\right)$, or multiple other sugars $\left.\left(\tau^{2}=1.72(0.37,1.48)\right)\right)$ within one category. In the fructose versus glucose comparison, six trials were responsible for the heterogeneity $\left(\tau^{2}=1.40\right.$ $(0.68,1.50))$. Three $[36,45,50]$ were subgroups of diabetic participants; they increased the magnitude of the mean difference. The other three trials $[32,33,46]$ showed important differences prior to the 2-hour time point (data not shown) but at two hours showed little or no difference between sweeteners. The single estimate of heterogeneity $\left(\tau^{2}\right)$ for the network meta-analysis was 0.65 (95\% CI 0.35,1.10).

Reporting the mixed evidence, two comparisons: fructose versus sucrose (MD $-1.12 \mathrm{mmol} / \mathrm{L}(-1.95,-0.27))$, and fructose versus glucose $(-1.56 \mathrm{mmol} / \mathrm{L}(-2.18,-1.02))$ were statistically significant, all favoring fructose, but neither of the confidence limits excluded the possibility of non-clinically relevant differences $(<1.15 \mathrm{mmol} / \mathrm{L}$ calculation based on a clinical important difference of 
Table 3 Mean difference in serum glucose $(\mathrm{mmol} / \mathrm{L})$ at 2 hours post-sweetener consumption and overnight fast in all participants

\begin{tabular}{|c|c|c|c|c|c|}
\hline $\begin{array}{c}\text { Non- } \\
\text { caloric } \\
0.05\end{array}$ & $\begin{array}{c}0.98 \\
(-1.24,3.25) \\
\tau^{2}=0.65(0.35,1.10)\end{array}$ & $\begin{array}{c}0.16 \\
(-1.46,1.80) \\
\text { consistent }\end{array}$ & $\begin{array}{c}1.19 \\
(-0.56,2.94)\end{array}$ & $\begin{array}{c}0.07 \\
(-1.45,1.61) \\
\text { consistent }\end{array}$ & $\begin{array}{c}-0.37 \\
(-2.07,1.29)\end{array}$ \\
\hline- & $\begin{array}{c}\text { Sugar } \\
\text { alcohols } \\
0.38\end{array}$ & $\begin{array}{c}-0.83 \\
(-2.66,1.03)\end{array}$ & $\begin{array}{c}0.21 \\
(-1.47,1.84)\end{array}$ & $\begin{array}{c}-0.93 \\
(-2.56,0.70) \\
\text { consistent }\end{array}$ & $\begin{array}{c}-1.37 \\
(-2.96,0.18) \\
\text { consistent }\end{array}$ \\
\hline $\begin{array}{c}-0.40 \\
(-0.79,-0.01) \\
N=1\end{array}$ & - & $\begin{array}{c}\text { Other } \\
\text { sugars } \\
0.01\end{array}$ & $\begin{array}{c}1.03 \\
(-0.13,2.20)\end{array}$ & $\begin{array}{c}-0.09 \\
(-1.00,0.81) \\
\text { consistent }\end{array}$ & $\begin{array}{c}-0.55 \\
(-1.61,0.50) \\
\text { consistent }\end{array}$ \\
\hline- & - & - & $\begin{array}{c}\text { Fructose } \\
0.55\end{array}$ & $\begin{array}{c}-1.12 \\
(-1.95,-0.27) \\
\text { consistent }\end{array}$ & $\begin{array}{c}-1.56 \\
(-2.18,-1.02) \\
\text { consistent }\end{array}$ \\
\hline $\begin{array}{c}0.30 \\
(-1.99,2.58) \\
N=\left.2\right|^{2}=0 \\
\tau^{2}=0\end{array}$ & $\begin{array}{c}0.41 \\
(-2.44,3.26) \\
N=1\end{array}$ & $\begin{array}{c}-0.28 \\
(-1.67,1.11) \\
N=7 \mathrm{I}^{2}=84 \\
\tau^{2}=1.72 \\
(0.37,1.48)\end{array}$ & $\begin{array}{c}-0.41 \\
(-1.30,0.47) \\
N=9 I^{2}=11 \\
\tau^{2}=0.17 \\
(0.58,2.41)\end{array}$ & $\begin{array}{c}\text { Sucrose/ } \\
\text { HFCS/ } \\
\text { Honey } \\
0\end{array}$ & $\begin{array}{c}-0.45 \\
(-1.15,0.21) \\
\text { consistent }\end{array}$ \\
\hline- & $\begin{array}{c}-2.20 \\
(-10.46,6.05) \\
N=3 I^{2}=85 \\
\tau^{2}=9.05 \\
(2.94,32.22)\end{array}$ & $\begin{array}{c}0.10 \\
(-2.46,2.66) \\
N=21^{2}=0 \\
\tau^{2}=0\end{array}$ & $\begin{array}{c}-\mathbf{1 . 4 0} \\
(-2.05,-0.74) \\
N=\left.23\right|^{2}=77 \\
\tau^{2}=1.4(0.68,1.50)\end{array}$ & $\begin{array}{c}-0.31 \\
(-0.53,-0.08) \\
N=\left.15\right|^{2}=0 \\
\tau^{2}=0 \\
(0,0.28)\end{array}$ & $\begin{array}{c}\text { Glucose } \\
0\end{array}$ \\
\hline
\end{tabular}

HFCS, high fructose corn syrup

The mixed evidence of the Bayesian network analysis are in the upper triangle and the direct evidence calculated using the REML estimate of $\tau^{2}$ are in the lower triangle. Sweeteners are reported in the expected order of efficacy[17] (with the exception of other sugars) from the expected lowest to highest 2-hour glucose response, with the estimated probability (or rank) listed in the diagonal. Each table cell contains the mean difference (MD) with the accompanying $95 \%$ confidence intervals. In the cells with direct evidence, we also list the number of studies, the $\mathrm{I}^{2}$ (percent of heterogeneity due to between-study heterogeneity) and $\tau^{2}$ (the between-study variance). Blank cells in the lower triangle indicate that no direct evidence was available. In the cells with mixed evidence, we list whether the mixed evidence was consistent with the available direct evidence. Also, in the first cell of the mixed evidence, we list the single $\tau^{2}$ estimate for the mixed evidence. Results are the MD of the expected higher-ranked sweeteners compared to the expected lower-ranked sweeteners (for example, MD of sugar alcohols versus sucrose is 0.41 and is in column 2, row 5 for the direct results, and is -0.93 and is in column 5 , row 2 for the network analysis results). MDs less than zero favor the expected higher-ranked sweetener (smaller glucose response). For example, sugar alcohols show an increased serum glucose response by $0.41 \mathrm{mmol} / \mathrm{L}$ compared to sucrose using the direct evidence. However, sugar alcohols show a decreased serum glucose response by $0.93 \mathrm{mmol} / \mathrm{L}$ using the mixed evidence. However, since both confidence intervals include zero, neither analysis allows a confident judgment about which sweetener is preferable. Pooled evidence significant at $P<0.05$ are presented in bold font. All nine mixed and direct results are consistent.

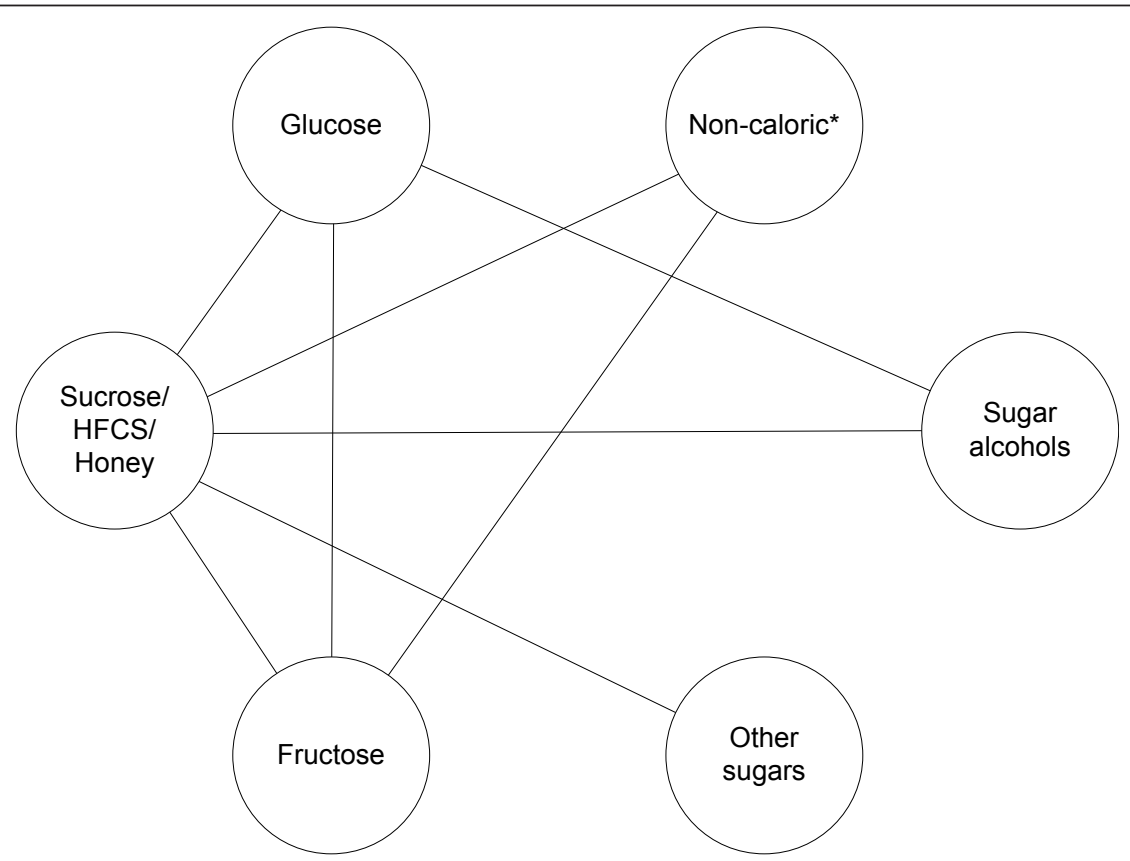

Figure 2 Network: blood glucose ( $\mathrm{mmol} / \mathrm{L})$ at $\mathbf{2}$ hours post-sweetener consumption and overnight fast. HFCS, high fructose corn syrup. *non-caloric sweetener groups were unavailable in the network with diabetic participants. 
$1 \%$ for $\mathrm{HbA1C}$ ) [60]. The weighted regression test for publication bias was not significant.

In the subnetwork of 31 trials enrolling participants without diabetes (446 participants; $\tau^{2}=3.66(1.66,7.31)$; Appendix Table 1 in Additional File 1), the direct evidence from all 8 comparisons was consistent with the mixed evidence from the network. The heterogeneity although reduced remained large between trials $\left(\mathrm{I}^{2}{ }^{\prime} \mathrm{s} \geq\right.$ $60 \%$ ) in both of the remaining multi-study direct evidence comparisons. Using the mixed evidence, three comparisons: fructose versus sucrose $(-0.54 \mathrm{mmol} / \mathrm{L}$ $(-1.06,-0.03))$, fructose versus glucose $(-0.89 \mathrm{mmol} / \mathrm{L}$ $(-1.21,-0.59))$, and fructose versus other sugars $(-0.85$ $\mathrm{mmol} / \mathrm{L}(-1.47,-0.21))$ were statistically significant, all favoring fructose, but none of the confidence limits excluded the possibility of non-clinically relevant differences.

In the subnetwork of ten trials enrolling participants with diabetes (152 participants; Appendix Table 2 in Additional File 1), the direct evidence from all six comparisons was consistent with the mixed evidence from the network. Note, this network did not include non-caloric sweeteners. Because the estimate of $\tau^{2}(224(0.14,139))$ did not converge, we report our findings from the direct evidence. Three direct comparisons were significant and found clinically relevant differences between agents over the entire confidence interval span: fructose versus glucose in 5 trials with 52 participants $\left(-4.81 \mathrm{mmol} / \mathrm{L}(-6 \cdot 34,-3 \cdot 29), \mathrm{I}^{2}=0 \%\right.$, $\left.\tau^{2}=0(0,7.47)\right)$, HSH versus glucose in 1 trial [29] with 12 participants $(-6.19 \mathrm{mmol} / \mathrm{L}(-9.78,-2.60))$ and isomaltulose versus sucrose in 1 trial [52] with 20 participants $(-3.44$ $\mathrm{mmol} / \mathrm{L}(-5 \cdot 31,-1 \cdot 56))$.

\section{Characteristics of trials studying effects on weight management, blood glucose and blood lipids}

Of the 13 trials (412 participants; Table 4), 3 trials compared a non-caloric sweetener (aspartame [61], cyclamate [62], or a mixture [63]) to sucrose, and 10 trials compared a saccharide to a different saccharide (glucose [64-66], fructose [64,65,67], mixtures of glucose and fructose [that is, sucrose [66-72] or honey [69]], FOS [71-73], a mixture of isomaltulose and sucrose [68], or tagatose [70]). No trials evaluated stevioside. Seven trials did not give any daily diet recommendations; one FOS trial recommended low-fiber intake [72]; one restricted added sweeteners to the assigned sweetener [62]; three trials restricted total energy levels and composition of macronutrients $(55 \%$ carbohydrate, $30 \%$ fat, $15 \%$ protein) $[64,65,67]$; and another restricted total energy levels and the composition to the assigned sweetener plus calcium caseinate [66]. With three exceptions $[63,64,66]$, the doses of sweeteners were at or below current clinical practice guideline $(\mathrm{CPG})$ recommendations (10\% of total energy intake (for example, $60 \mathrm{~g}$ of sucrose in a $2000 \mathrm{kcal}$ diet) although only three trials $[64,65,67]$ restricted overall energy intake, therefore further sweetener consumption may have exceeded current recommendations. One trial [63] prescribed sweeteners (simple carbohydrates) at $25 \%$ of total energy intake the American Diabetes Association (ADA) 2004 recommended maximum. The earliest trial [66] prescribed sweeteners at $87 \%$ of total energy intake - they were differentiating energy availability from energy content.

Four trials were in diabetic populations [62,67,71,73], seven trials were in generally healthy populations [61,64-66,68,70,72] and two trials were in overweight/ obese [69] or overweight [63] populations. Mean BMI levels ranged from 21 to $31 \mathrm{~kg} / \mathrm{m}^{2}$. Median mean age was 35 years and median sex distribution was $54 \%$ women.

Sample size ranged from 10 to 133 (median 20), 1 had a sample size $\geq 30$ per group and duration of follow-up ranged from 1 to 12 weeks (median 4 weeks). Ten were crossover trials [62,64-67,70-73] and four were parallel trials $[61,63,68,69]$. Jadad scores ranged from 1 to 2 (median 1). Twelve of thirteen trials did not report whether or how treatment assignment was concealed. One used alternating assignments according to body weight [70].

\section{Non-caloric versus saccharide: effects on weight management, blood glucose and blood lipids}

Two trials reported change in BMI (Table 5). The 4week trial in healthy participants [61] did not find a significant loss in BMI in non-caloric sweetener recipients $\left(-0.3 \mathrm{~kg} / \mathrm{m}^{2}(-1.1,0.5), 133\right.$ participants). The trial in overweight participants [63] found a significantly greater loss in BMI over ten weeks of follow-up in participants consuming the non-caloric sweetener $\left(-0.9 \mathrm{~kg} / \mathrm{m}^{2}(-1.5,-\right.$ $0.4), 41$ participants). Two trials reported absolute change in weight. One crossover trial was done in type 1 diabetic participants and found no difference in weight loss between groups over four weeks $(0.8 \mathrm{~kg}(-3.3,4.9)$, ten participants [62]). The other trial in overweight participants [63] found significantly greater weight loss over 10 weeks in the non-caloric sweetener group $(-2.6 \mathrm{~kg}$ $(-3.7,-1.5), 41$ participants).

Two trials reported energy intake; both reported a significant effect of non-caloric sweeteners. The 4-week trial in generally healthy participants [61] found a significantly reduced intake of calories in non-caloric sweetener participants $(-283 \mathrm{kcal}(-414,-153), 133$ participants). The trial in overweight participants [63] also found significantly less energy intake (over one day) in the non-caloric sweetener group after ten weeks of follow-up (-491 kcal (-806,-177), 41 participants).

Available trials found no effect of sweetener type on HbA1C (one trial: $-0.02 \%$ over four weeks $(-0.40,0.30)$, 
Table 4 Characteristics of included randomized trials with effects on weight management, blood glucose and blood lipids

\begin{tabular}{|c|c|c|c|c|c|c|c|}
\hline $\begin{array}{l}\text { Author, } \\
\text { Year, } \\
\text { Country }\end{array}$ & $\begin{array}{l}\text { Population, } \\
\text { Mean BMI } \\
\left(\mathrm{kg} / \mathrm{m}^{2}\right)\end{array}$ & $\begin{array}{c}\text { Sweetener 1: } \\
\text { Type, } \\
\text { Quantity (g/d) }\end{array}$ & $\begin{array}{l}\text { Sweetener 2: } \\
\text { Type, } \\
\text { Quantity (g/ } \\
\text { d) }\end{array}$ & $\begin{array}{c}\text { Daily Diet } \\
\text { (carbohydrate/ } \\
\text { fat/protein) }\end{array}$ & $\begin{array}{c}\text { Study } \\
\text { design, } \\
\text { Follow-up, } \\
\text { Sample } \\
\text { size }\end{array}$ & $\begin{array}{c}\text { Mean age } \\
(y), \\
\% \text { female }\end{array}$ & $\begin{array}{c}\text { Allocation } \\
\text { concealment, } \\
\text { Jadad score, } \\
\text { Funding }\end{array}$ \\
\hline \multicolumn{8}{|c|}{ Non-caloric versus Saccharide } \\
\hline $\begin{array}{l}\text { Reid [61] } \\
2007 \\
\text { UK }\end{array}$ & $\begin{array}{c}\text { General } \\
23\end{array}$ & $\begin{array}{c}\text { Aspartame } \\
3.56\end{array}$ & $\begin{array}{c}\text { Sucrose } \\
42\end{array}$ & Ad lib & $\begin{array}{l}\mathrm{RCT} \\
4 \mathrm{wk} \\
133\end{array}$ & $\begin{array}{c}32 \\
100\end{array}$ & $\begin{array}{l}\text { Unclear } \\
1 \\
\text { Public }\end{array}$ \\
\hline $\begin{array}{l}\text { Raben [63] } \\
2002 \\
\text { Denmark }\end{array}$ & $\begin{array}{c}\text { Overweight } \\
28\end{array}$ & $\begin{array}{c}\text { Aspartame/ } \\
\text { Acesulfame/ } \\
\text { Cyclamate/ } \\
\text { Saccharin } \\
0.48-0.67\end{array}$ & $\begin{array}{l}\text { Sucrose } \\
125-175\end{array}$ & Ad lib & $\begin{array}{l}\text { RCT } \\
10 w k \\
41\end{array}$ & $\begin{array}{l}35 \\
85\end{array}$ & $\begin{array}{c}\text { Unclear } \\
1 \\
\text { Mixed }^{\text {a }}\end{array}$ \\
\hline $\begin{array}{c}\text { Chantelau [62] } \\
1985 \\
\text { Germany }\end{array}$ & $\begin{array}{l}\mathrm{DM} 1 \\
<25\end{array}$ & $\begin{array}{c}\text { Cyclamate } \\
348 \mathrm{mg}\end{array}$ & $\begin{array}{c}\text { Sucrose } \\
24\end{array}$ & $\begin{array}{l}\text { Restricted to no other } \\
\text { added sweeteners however } \\
\text { sucrose-sweetened soft } \\
\text { drinks were discouraged }\end{array}$ & $\begin{array}{l}x R C T \\
24 w k \\
\text { periods } \\
10\end{array}$ & $\begin{array}{l}\text { Range } 25- \\
43 \\
80\end{array}$ & $\begin{array}{c}\text { Unclear } \\
1 \\
-\end{array}$ \\
\hline \multicolumn{8}{|c|}{ Saccharide versus Saccharide } \\
\hline $\begin{array}{l}\text { Okuno [68] } \\
2010 \\
\text { Japan }\end{array}$ & $\begin{array}{c}\text { General } \\
23\end{array}$ & $\begin{array}{c}\text { Isomaltulose/ } \\
\text { Sucrose } \\
40\end{array}$ & $\begin{array}{l}\text { Sucrose } \\
\quad 40\end{array}$ & Ad lib & $\begin{array}{l}\text { RCT } \\
12 \mathrm{wk} \\
50\end{array}$ & $\begin{array}{l}53 \\
80\end{array}$ & $\begin{array}{l}\text { Unclear } \\
2 \\
\text { Private }\end{array}$ \\
\hline $\begin{array}{l}\text { Tudor Ngo } \\
\text { Sock [64] } \\
2010 \\
\text { Netherlands }\end{array}$ & $\begin{array}{l}\text { General } \\
19-25\end{array}$ & $\begin{array}{c}\text { Fructose } \\
3.5 \mathrm{~g} / \mathrm{kg} \text { FFM }\end{array}$ & $\begin{array}{c}\text { Glucose } \\
3.5 \mathrm{~g} / \mathrm{kg} \text { FFM }\end{array}$ & $\begin{array}{c}\text { Total and distribution of } \\
\text { energy restricted } \\
55 / 30 / 15 \%\end{array}$ & $\begin{array}{c}\text { xRCT } \\
21 w k \\
\text { periods } \\
\text { (2-3wk wo) } \\
11\end{array}$ & $\begin{array}{c}25 \\
0\end{array}$ & $\begin{array}{l}\text { Unclear } \\
1 \\
\text { Mixed }\end{array}$ \\
\hline $\begin{array}{c}\text { Yaghoobi [69] } \\
2008 \\
\text { Iran }\end{array}$ & $\begin{array}{l}\text { Overweight/ } \\
\text { Obese } \\
31\end{array}$ & $\begin{array}{l}\text { Honey } \\
70\end{array}$ & $\begin{array}{c}\text { Sucrose } \\
70\end{array}$ & Ad lib & $\begin{array}{c}\text { RCT } \\
\text { Max 30d } \\
55\end{array}$ & $\begin{array}{l}42 \\
56\end{array}$ & $\begin{array}{c}\text { Unclear } \\
1 \\
\text { Public }\end{array}$ \\
\hline $\begin{array}{l}\text { Boesch [70] } \\
2001 \\
\text { Switzerland }\end{array}$ & $\begin{array}{l}\text { General } \\
<25\end{array}$ & $\begin{array}{c}\text { Tagatose } \\
45\end{array}$ & $\begin{array}{c}\text { Sucrose } \\
45\end{array}$ & Ad lib & $\begin{array}{c}\text { xRCT } \\
228 d \\
\text { periods } \\
\text { (28d wo) } \\
12\end{array}$ & $\begin{array}{c}\text { Range } 21- \\
30 \\
0\end{array}$ & $\begin{array}{c}\text { Inadequate } \\
2 \\
-\end{array}$ \\
\hline $\begin{array}{l}\text { Bantle [65] } \\
2000 \\
\text { US }\end{array}$ & $\begin{array}{l}\text { General } \\
25\end{array}$ & $\begin{array}{l}\text { Fructose } \\
80 \\
\text { (incl } 17 \mathrm{~g} \\
\text { glucose) }\end{array}$ & $\begin{array}{l}\text { Glucose } \\
\quad 80 \\
\text { (incl } 15 \mathrm{~g} \\
\text { fructose) }\end{array}$ & $\begin{array}{l}\text { Total and distribution of } \\
\text { energy restricted } \\
55 / 30 / 15 \%\end{array}$ & $\begin{array}{l}x R C T \\
242 d \\
\text { periods } \\
24\end{array}$ & $\begin{array}{l}41 \\
50\end{array}$ & $\begin{array}{l}\text { Unclear } \\
1 \\
\text { Public }\end{array}$ \\
\hline $\begin{array}{l}\text { Luo [71] } \\
2000 \\
\text { Belgium }\end{array}$ & $\begin{array}{c}\mathrm{DM} 2 \\
28\end{array}$ & $\begin{array}{l}\text { FOS } \\
20\end{array}$ & $\begin{array}{c}\text { Sucrose } \\
20\end{array}$ & Ad lib & $\begin{array}{c}\text { xRCT } \\
24 w k \\
\text { periods } \\
\text { (2wk wo) } \\
10\end{array}$ & $\begin{array}{l}57 \\
40\end{array}$ & $\begin{array}{c}\text { Unclear } \\
1 \\
\text { Private }\end{array}$ \\
\hline $\begin{array}{c}\text { Alles [73] } \\
1999 \\
\text { Netherlands }\end{array}$ & $\begin{array}{c}\mathrm{DM} 2 \\
28\end{array}$ & $\begin{array}{c}\text { FOS } \\
\text { Saccharide } \\
30\end{array}$ & $\begin{array}{c}\text { Glucose } \\
\text { Saccharide } \\
8\end{array}$ & Ad lib & $\begin{array}{l}x R C T \\
220 d \\
\text { periods } \\
20\end{array}$ & $\begin{array}{l}59 \\
55\end{array}$ & $\begin{array}{c}\text { Unclear } \\
1 \\
\text { Mixed }\end{array}$ \\
\hline $\begin{array}{l}\text { Luo [72] } \\
1996 \\
\text { France }\end{array}$ & $\begin{array}{c}\text { General } \\
21\end{array}$ & $\begin{array}{l}\text { FOS } \\
20\end{array}$ & $\begin{array}{c}\text { Sucrose } \\
20\end{array}$ & $\begin{array}{l}\text { Low-fiber diet } \\
\text { recommended }\end{array}$ & $\begin{array}{c}x R C T \\
24 w k \\
\text { periods } \\
\text { (2wk wo) } \\
12\end{array}$ & $\begin{array}{c}24 \\
0\end{array}$ & $\begin{array}{l}\text { Unclear } \\
2 \\
\text { Private }\end{array}$ \\
\hline $\begin{array}{c}\text { Bantle [67] } \\
1986 \\
\text { US }\end{array}$ & $\begin{array}{c}\text { 50\% } \mathrm{DM} 1 / 50 \% \\
\mathrm{DM} 2 \\
-\end{array}$ & $\begin{array}{c}\text { Sucrose } \\
23 \%\end{array}$ & $\begin{array}{c}\text { Fructose } \\
21 \%\end{array}$ & $\begin{array}{c}\text { Total and distribution of } \\
\text { energy restricted } \\
55 / 30 / 15 \%\end{array}$ & $\begin{array}{c}x R C T \\
28 d \\
\text { periods } \\
24\end{array}$ & $\begin{array}{l}43 \\
54\end{array}$ & $\begin{array}{c}\text { Unclear } \\
1 \\
\text { Mixed }\end{array}$ \\
\hline $\begin{array}{c}\text { Macdonald [66] } \\
1973 \\
\text { UK }\end{array}$ & General & $\begin{array}{l}\text { Sucrose } \\
6.5 \mathrm{~g} / \mathrm{kg}\end{array}$ & $\begin{array}{l}\text { Glucose } \\
6.5 \mathrm{~g} / \mathrm{kg}\end{array}$ & $\begin{array}{l}\text { Restricted to } \\
1 \mathrm{~g} / \mathrm{kg} \text { calcium } \\
\text { caseinate }\end{array}$ & $\begin{array}{c}\text { xRCT } \\
211 \mathrm{~d} \\
\text { periods } \\
(2 \mathrm{wk} \text { wo }) \\
10\end{array}$ & $\begin{array}{l}\text { Range } 20- \\
25 \\
40\end{array}$ & $\begin{array}{c}\text { Unclear } \\
1 \\
\text { Private }\end{array}$ \\
\hline
\end{tabular}

UK, United Kingdom; US, United States; DM1, type 1 diabetes mellitus; DM2, type 2 diabetes mellitus; HFCS, high fructose corn syrup; FOS, fructooligosaccharide; FFM, fat free mas; $x$ RCT, controlled crossover trial; RCT, parallel randomized controlled trial; wo, washout; max, maximum; "-" means the value was not reported in the study

${ }^{\mathrm{a} B o t h}$ public and private sources of funding 
Table 5 Weight management, blood glucose and blood lipids: Non-caloric versus Sucrose

\begin{tabular}{|c|c|c|c|c|}
\hline $\begin{array}{l}\text { Non-caloric } \\
\text { sweetener }\end{array}$ & Population & Timepoint (week) & No of participants & $\mathrm{MD}(95 \% \mathrm{Cl})$ \\
\hline \multicolumn{5}{|c|}{ BMI, $\mathrm{kg} / \mathrm{m}^{2}$} \\
\hline Aspartame & General & 4 & 133 & $-0.3(-1.1,0.5)$ \\
\hline Mixture $^{a}$ & Overweight & 10 & 41 & $-0.9(-1.5,-0.4)$ \\
\hline \multicolumn{5}{|c|}{ Weight, kg } \\
\hline Cyclamate & DM1 & 4 & 10 & $0.8(-3.3,4.9)$ \\
\hline Mixture & Overweight & 10 & 41 & $-2.6(-3.7,-1.5)$ \\
\hline \multicolumn{5}{|c|}{ Day Energy Intake, kcal } \\
\hline Aspartame & General & 4 & 133 & $-283(-414,-153)$ \\
\hline Mixture & Overweight & 10 & 41 & $-491(-806,-177)$ \\
\hline \multicolumn{5}{|c|}{$\mathrm{HbA} 1 \mathrm{C}, \%$} \\
\hline Cyclamate & DM1 & 4 & 10 & $-0.02(-0.4,0.3)$ \\
\hline \multicolumn{5}{|c|}{ HOMA Index } \\
\hline Mixture & Overweight & 10 & 41 & $-0.20(-0.58,0.18)$ \\
\hline \multicolumn{5}{|c|}{ Total Cholesterol, $\mathrm{mmol} / \mathrm{L}$} \\
\hline Cyclamate & DM1 & 4 & 10 & $-0.34(-0.87,0.19)$ \\
\hline \multicolumn{5}{|c|}{ HDL Cholesterol, $\mathrm{mmol} / \mathrm{L}$} \\
\hline Cyclamate & DM1 & 4 & 10 & $-0.05(-0.32,0.22)$ \\
\hline \multicolumn{5}{|c|}{ Triglycerides, mmol/L } \\
\hline Cyclamate & DM1 & 4 & 10 & $-0.02(-0.16,0.12)$ \\
\hline Mixture & Overweight & 10 & 41 & $-0.26(-0.85,0.34)$ \\
\hline
\end{tabular}

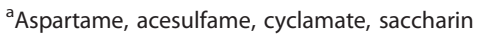

DM1, Type 1 Diabetes Mellitus; DM2, Type 2 Diabetes Mellitus; BMI, Body mass index; HbA1C, Glycated haemoglobin; HOMA, Homeostatic Model Assessment; $\mathrm{MD}$, Mean difference; $\mathrm{Cl}$, Confidence interval

Statistically significant results are bolded.

ten participants [62]) or the HOMA index (one trial: -0.20 over ten weeks $(-0.58,0.18)$, forty-one participants [63]). The trial in ten type 1 diabetic participants [62] found no effect on total cholesterol, HDL cholesterol, or triglycerides over the course of four weeks; the other trial in forty-one overweight participants [63] found no effect on triglycerides over the course of ten weeks.

\section{Saccharide versus saccharide: effects on weight management, blood glucose and blood lipids}

Two trials reported change in BMI (Table 6); one comparing honey to sucrose in overweight/obese participants over 4 weeks of follow-up [69]; the other comparing a mixture of isomaltulose and sucrose to sucrose over 12 weeks of follow-up [68] in healthy participants. Neither found a significant difference between sweeteners. One trial compared FOS to glucose [73] (three weeks in twenty diabetic participants) and one trial compared FOS to sucrose [72] (four weeks in twelve healthy participants), respectively. Neither found a difference in absolute weight change. Five other trials done in varying populations (including overweight/obese [69] or healthy populations [64-66,68]) found no differences in change in absolute weight between sweeteners.
Two trials reported energy intake (FOS compared with glucose [73] and sucrose [72] respectively, but neither found a significant difference.

Two trials (one comparing FOS to sucrose [71] and one comparing isomaltulose/sucrose to sucrose [68]) found no significant effect on HbA1C. However, the latter [68] found a significant decrease in the HOMA index among isomaltulose/sucrose recipients $(-0.44$ $(-0.76,-0.12))$.

Seven trials reported change in total cholesterol. The pooled result of two trials [71,72] comparing FOS to sucrose was statistically significant $(0.26 \mathrm{mmol} / \mathrm{L}$ $\left.(0.03,0.48), \mathrm{I}^{2}=0 \%, \tau^{2}=0(0,0.01)\right)$, although this conclusion was based on a total of only twenty-two participants. One trial comparing isomaltulose and sucrose to sucrose (50 healthy participants over 12 weeks) [68] found a significantly smaller increase in total cholesterol for the isomaltulose/sucrose group (-0.10 $\mathrm{mmol} / \mathrm{L}(-0.17,-0.02))$. No trials found an effect of sweetener type on LDL cholesterol or HDL cholesterol. The trial comparing isomaltulose and sucrose to sucrose [68] also found a significant effect on triglycerides $(-0.27 \mathrm{mmol} / \mathrm{L}(-0.44,-0.10), 0.11$ decrease versus $0.16 \mathrm{mmol} / \mathrm{L}$ increase). However, four trials studying other combinations of sweeteners 
Table 6 Weight management, blood glucose and blood lipids: Saccharide vs Saccharide

\begin{tabular}{|c|c|c|c|c|}
\hline Comparison & Population & Timepoint (week) & No of participants & MD $(95 \% \mathrm{Cl})$ \\
\hline \multicolumn{5}{|c|}{ BMI, $\mathrm{kg} / \mathrm{m}^{2}$} \\
\hline Honey vs Sucrose & Overweight/Obese & 4 & 55 & $-0.5(-3.1,2.1)$ \\
\hline Isomaltulose/Sucrose vs Sucrose & General & 12 & 50 & $-0.04(-0.4,0.3)$ \\
\hline \multicolumn{5}{|c|}{ Weight, kg } \\
\hline Fructose vs Glucose & General & 6 & 24 & $0.1(-3.4,3.6)$ \\
\hline Fructose vs Glucose & General & 1 & 11 & $-0.4(-3.1,2.3)$ \\
\hline FOS vs Glucose & DM2 & 3 & 20 & $0.2(-5.2,5.6)$ \\
\hline FOS vs Sucrose & General & 4 & 12 & $1.0(-2.4,4.4)$ \\
\hline Honey vs Sucrose & Overweight/Obese & 4 & 55 & $-1.5(-6.9,3.9)$ \\
\hline Isomaltulose/Sucrose vs Sucrose & General & 12 & 50 & $-0.06(-0.9,0.8)$ \\
\hline Sucrose vs Glucose & General & 2 & 10 & $0.2(-0.07,0.4)$ \\
\hline \multicolumn{5}{|c|}{ Energy Intake, kcal } \\
\hline FOS vs Glucose & DM2 & 3 & 20 & $-139(-399,122)$ \\
\hline FOS vs Sucrose & General & 4 & 12 & $-56(-156,43)$ \\
\hline \multicolumn{5}{|c|}{$\mathrm{HbA} 1 \mathrm{C}, \%$} \\
\hline FOS vs Sucrose & DM2 & 4 & 10 & $0.17(-0.59,0.93)$ \\
\hline Isomaltulose/Sucrose vs Sucrose & General & 12 & 50 & $0.01(-0.05,0.07)$ \\
\hline \multicolumn{5}{|c|}{ HOMA Index } \\
\hline Isomaltulose/Sucrose vs Sucrose & General & 12 & 50 & $-0.44(-0.76,-0.12)$ \\
\hline \multicolumn{5}{|c|}{ Total Cholesterol, $\mathrm{mmol} / \mathrm{L}$} \\
\hline Fructose vs Glucose & General & 1 & 11 & $0.10(-0.24,0.44)$ \\
\hline FOS vs Glucose & DM2 & 3 & 20 & $0.20(-0.27,0.67)$ \\
\hline FOS vs Sucrose & DM2 & 4 & 10 & $0.15(-0.24,0.54)$ \\
\hline FOS vs Sucrose & General & 4 & 12 & $0.31(0.03,0.59)$ \\
\hline Honey vs Sucrose & Overweight/Obese & 4 & 55 & $-0.11(-0.26,0.05)$ \\
\hline Isomaltulose/Sucrose vs Sucrose & General & 12 & 50 & $-0.10(-0.17,-0.02)$ \\
\hline Tagatose vs Sucrose & General & 4 & 12 & $-0.11(-0.51,0.29)$ \\
\hline \multicolumn{5}{|c|}{ LDL Cholesterol, $\mathrm{mmol} / \mathrm{L}$} \\
\hline Fructose vs Glucose & General & 1 & 11 & $0(-0.17,0.17)$ \\
\hline FOS vs Sucrose & DM2 & 4 & 10 & $0.13(-0.21,0.47)$ \\
\hline Honey vs Sucrose & Overweight/Obese & 4 & 55 & $-0.03(-0.22,0.16)$ \\
\hline Isomaltulose/Sucrose vs Sucrose & General & 12 & 50 & $-0.02(-0.08,0.04)$ \\
\hline Tagatose vs Sucrose & General & 4 & 12 & $0.09(-0.26,0.44)$ \\
\hline \multicolumn{5}{|c|}{$\mathrm{HDL}$ Cholesterol, $\mathrm{mmol} / \mathrm{L}$} \\
\hline Fructose vs Glucose & General & 1 & 11 & $0(-0.17,0.17)$ \\
\hline FOS vs Sucrose & General & 4 & 12 & $-0.06(-0.14,0.02)$ \\
\hline FOS vs Sucrose & DM2 & 4 & 10 & $0.07(-0.03,0.17)$ \\
\hline Honey vs Sucrose & Overweight/Obese & 4 & 55 & $0.01(-0.12,0.14)$ \\
\hline Isomaltulose/Sucrose vs Sucrose & General & 12 & 50 & $-0.02(-0.05,0.01)$ \\
\hline Tagatose vs Sucrose & General & 4 & 12 & $-0.17(-0.28,0.06)$ \\
\hline \multicolumn{5}{|c|}{ Triglycerides, $\mathrm{mmol} / \mathrm{L}$} \\
\hline FOS vs Glucose & DM2 & 3 & 20 & $0.12(-0.30,0.54)$ \\
\hline FOS vs Sucrose & General & 4 & 12 & $0.18(-0.03,0.39)$ \\
\hline FOS vs Sucrose & DM2 & 4 & 10 & $-0.18(-0.38,0.02)$ \\
\hline Honey vs Sucrose & Overweight/Obese & 4 & 55 & $-0.10(-0.22,0.02)$ \\
\hline Isomaltulose vs Sucrose & General & 12 & 50 & $-0.27(-0.44,-0.10)$ \\
\hline
\end{tabular}

*Aspartame, acesulfame, cyclamate, saccharin

DM1 Type 1 Diabetes Mellitus, DM2 Type 2 Diabetes Mellitus, BMI Body mass index, HbA1C Glycated hemoglobin, HOMA Homeostatic Model Assessment, MD Mean difference, $\mathrm{Cl}$ Confidence interval

Statistically significant results are bolded. 
[69,71-73] found no effect of sweetener choice on triglyceride levels.

\section{Discussion}

To our knowledge, this is the first systematic review of randomized trial evidence that examines comparative sweetener effectiveness in diabetic, overweight/obese, and healthy populations. Despite tremendous interest in hypocaloric sweeteners as a potential tool to prevent obesity and its complications, we found little evidence to support their health benefits as compared to caloric alternatives. Based on analyses of two trials, we found that the inclusion of non-caloric sweeteners in the diet resulted in reduced energy intake compared to the caloric (sucrose) groups - approximately $500 \mathrm{kcal} /$ day less over 10 weeks or $250 \mathrm{kcal} /$ day over 4 weeks. The longer of these trials found that those in the non-caloric sweetener group also had a decrease in BMI compared to an increase in BMI in the sucrose group $(-0.40$ versus 0.50 $\mathrm{kg} / \mathrm{m}^{2}$, and -1.00 versus $1.60 \mathrm{~kg}$, respectively) [63]. Given that the control group was asked to ingest supplemental calories in addition to their regular ad lib diet, a BMI reduction of approximately $1 \mathrm{~kg} / \mathrm{m}^{2}$ over 10 weeks (or $0.1 \mathrm{~kg} / \mathrm{m}^{2} /$ week) may be overly optimistic. However, even a reduction in BMI of $0.05 \mathrm{~kg} / \mathrm{m}^{2} /$ week would be clinically relevant if sustained for a year or more. The remaining analyses comparing non-caloric and caloric sweeteners were non-significant.

\section{Main findings}

- 53 randomized controlled trials were included - all small and largely short-term (only 13 trials with $\geq 1$ week durations)

- 2-hour blood glucose (mixed evidence, $\tau^{2}=3.66$ (95\% CI 1.66,7.31): fructose versus sucrose (MD $-0.54 \mathrm{mmol} / \mathrm{L}(-1.06,-0.03))$, fructose versus glucose $(-0.89 \mathrm{mmol} / \mathrm{L}(-1.21,-0.59))$, fructose versus other sugars $(-0.85 \mathrm{mmol} / \mathrm{L}(-1.47,-0.21))$ in non-diabetic participants

- 2-hour blood glucose (direct evidence): fructose versus glucose $\left(-4.81 \mathrm{mmol} / \mathrm{L}(-6.34,-3.29), \mathrm{I}^{2}=0 \%\right.$, $\tau^{2}=0$ (0,7.47), 5 trials in 52 diabetic participants)

- change in BMI: non-caloric mixture versus sucrose (MD $-0.9 \mathrm{~kg} / \mathrm{m}^{2}[-1.5,-0.4]$, in 41 overweight participants, over 10 weeks), non-caloric aspartame versus sucrose $\left(-0.3 \mathrm{~kg} / \mathrm{m}^{2}(-1 \cdot 1,0 \cdot 5), 133\right.$ healthy participants, over 4 weeks)

- energy intake (over one day): non-caloric aspartame versus sucrose $(-283 \mathrm{kcal}(-414,-153), 133$ healthy participants, over 4 weeks), non-caloric mixture versus sucrose $(-491 \mathrm{kcal}(-806,-177), 41$ overweight participants, over 10 weeks)

- total cholesterol: FOS versus sucrose $(0.26 \mathrm{mmol} / \mathrm{L}$ $(0.03,0.48), \mathrm{I}^{2}=0 \%, \tau^{2}=0(0,0.01), 2$ trials with a total of 12 healthy and 10 type 2 diabetic participants, over 4 weeks)

Head-to-head comparisons between saccharides did not identify any statistically significant differences. The confidence limits of these results either included minimally important differences or the group sizes were too small $(<30)$ to have good estimates of standard deviation [74]. The one exception was the comparison between sucrose and FOS, which suggested that total cholesterol was reduced to a greater extent with sucrose than with FOS. However, the confidence intervals for this analysis included values that were not clinically relevant $(0.03$ to $0.59 \mathrm{mmol} / \mathrm{L})$. There was no evidence that HFCS or fructose increased levels of cholesterol relative to other sweeteners.

Although we found that fructose reduced 2-hour blood glucose concentrations by $4.81 \mathrm{mmol} / \mathrm{L}$ compared to glucose in diabetic participants, data comparing noncaloric and sugar alcohols to the more commonly used sucrose or HFCS were inconclusive. Contrary to perception and current recommendations, no substantive evidence describing important long-term benefits of hypocaloric sweeteners for diabetic patients were identified. Also, despite popular belief, no high-quality RCT evidence was found indicating that fructose causes or exacerbates hypertriglyceridemia [6].

Although the identified trials were numerous, they were very small and largely short-term. We found 13 trials with participant follow-up longer than 1 week and group sizes $\geq 10$ : 3 that compared non-caloric sweeteners to sucrose, and 10 that were head-to-head comparisons of saccharides. Ten of 13 trials had a Jadad score of 1 and none adequately concealed treatment assignment prior to assignment. Although blinding the participants would have been impossible in many of the trials due to taste differences between sweeteners [63], the reporting of important design descriptors were largely absent, indicating a substantial risk for bias $[12,13]$. The longest trial was only 10 weeks - not long enough to determine whether substituting a non-caloric sweetener for a caloric sweetener is sustainable in daily practice. To detect an important reduction in weight over at least one year such as $2.5 \mathrm{~kg} / \mathrm{m}^{2}$ (less than $0.05 \mathrm{~kg} / \mathrm{m}^{2}$ / week) in a RCT would require a minimum of 85 participants (assumptions: 25\% loss-to-follow-up, $\alpha=0.05$, power $\left.=90 \%, \mathrm{SD}=3 \mathrm{~kg} / \mathrm{m}^{2}\right)$.

Our network meta-analysis had several limitations: 1) the sugar alcohol and other sugar categories contained multiple sweeteners that are likely to have different blood glucose profiles thereby inducing heterogeneity, 2) power to detect inconsistency is limited by the number of trials included in each test, and 3) the back-calculation method used to detect inconsistency involved 
multiple tests thereby increasing the false-positive rate. However, we did not detect any inconsistency.

Another limitation was that only three studies restricted the total energy consumed by each participant. Therefore, participants may have supplemented energy lost with non-caloric sweeteners with other food products - sweetened or otherwise. However, it may be argued that this is a strength of the trials - in that they reflect what happens in real world self-management diet practices. Lastly, and perhaps most importantly, all studies were small, thereby underestimating standard deviation and as a result underestimating confidence interval widths and increasing the likelihood of false-positive findings [74]. Despite this, the confidence intervals for many analyses were wide and did not exclude a minimally important difference. Small study bias (or publication bias) may also play a role in our findings concerning longer-term outcomes.

In theory, substituting non-caloric and lower caloric sweeteners for simple sugars should reduce energy intake and thereby the risk of obesity and its consequences. However, there are a number of reasons why increasing use of non-caloric and lower caloric sweeteners might not lead to the expected improvements in energy regulation. First, use of hypocaloric sweeteners might not induce weight loss even in the short term. For example, if reductions in calories due to sweeteners are offset by increases in caloric intake from other sources $[75,76]$, or offset by decreases in caloric expenditure $[77,78]$. Although our data suggest that non-caloric sweeteners may lead to clinically relevant weight loss through reduced energy consumption, this conclusion was driven by a single trial with a total of 41 participants. Unlike caloric sweeteners (which may partially compensate added calories with reduced energy intake from other sources) [79], non-caloric sweeteners are not known to suppress appetite, and therefore would not reduce the motivation to eat. Furthermore, it has been suggested that the psychobiological signals with noncaloric sweeteners may directly influence physiological regulatory mechanisms and thus further reduce their potential for reducing net energy intake $[75,80]$. Second, if calorie reduction is not maintained, short-term reductions in weight due to the use of hypocaloric sweeteners might not be sustained. Third, it is possible though speculative that any health benefits due to weight loss from non-caloric sweeteners might be wholly or partially offset by currently unrecognized adverse events due to their use. The lack of data on the long-term benefits of non-caloric sweeteners means that it is currently impossible to determine whether these substances will improve public health.

\section{Conclusions}

In summary, despite the public health importance of obesity, and obesity-related chronic diseases (for example, diabetes); the clear role of excessive caloric intake in these conditions; and the billions of dollars spent on non-caloric sweeteners [4,5], little high-quality clinical research has been done to identify the potential harms and benefits of hypocaloric sweeteners. Since even small reductions (as little as 6\%) in body-weight can prevent chronic disease [81,82], hypocaloric sweeteners could play an important role in a wider population health strategy to prevent, reduce and manage obesity-related comorbidities. Eliminating unnecessary added sweeteners from food products (for example, buns, crackers, and processed meats) and substituting sugars with lower calorie sweeteners in foods such as desserts and drinks could significantly improve health. Long-term, highquality, adequately powered randomized controlled trials are required to confirm this hypothesis by assessing the clinically relevant outcomes reported in this review.

\section{Abbreviations list}

ADA: American Diabetes Association; ADI: Acceptable Daily Intake; BMI: body mass index; CPG: Clinical Practice Guideline; FOS: fructooligosaccharide; HbA1C: glycated haemoglobin; HDL: high density lipoprotein; HFCS: high fructose corn syrup; HOMA: Homeostatic Model Assessment; LDL: low density lipoprotein; MCMC: Markov chain Monte Carlo; MD: mean difference; RCT: randomized controlled trial; SD: standard deviation.

\section{Additional material}

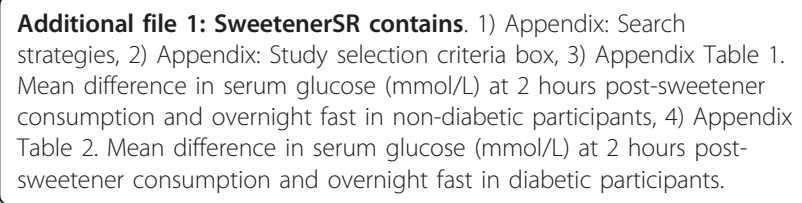

\section{Acknowledgements}

The authors of this report are grateful to Dale Storie for librarian support and to Natasha Krahn, Nicola Hooton, and Sophanny Tiv for additional reviewer support, and Ghenette Houston for administrative support. Support for this work was provided in part through an Interdisciplinary Team Grant to the Interdisciplinary Chronic Disease Collaboration from the Alberta Heritage Foundation for Medical Research (AHFMR). AHFMR was not involved in the interpretation of results or the drafting of the manuscript.

\section{Author details}

'Department of Medicine, 13-103 Clinical Sciences Building, University of Alberta, Edmonton, Alberta, T6G 2G3 Canada. ${ }^{2}$ Department of Agricultural, Food \& Nutritional Science, 410 Agriculture/Forestry Centre, University of Alberta, Edmonton, Alberta, T6G 2P5 Canada. ${ }^{3}$ Department of Pediatrics, 
8213 Aberhart Centre, University of Alberta, Edmonton, Alberta, T6G 2J3 Canada.

\section{Authors' contributions}

NW conceived the study, did the statistical analyses and wrote the first draft of the manuscript with assistance from MT. All authors contributed to the design, interpretation of results and critical revision of the article for intellectually important content. All authors read and approved the final manuscript.

\section{Competing interests}

Dr. Tonelli has received a peer reviewed Canadian Institutes of Health Research Industry Partnership Operating Grant, from Abbott Laboratories. Dr. Field is a scientific advisor to International Life Science Institute North America (non-financial arrangement).

Received: 14 June 2011 Accepted: 17 November 2011

Published: 17 November 2011

\section{References}

1. Parker KJ: Alternatives to sugar. The search for an ideal non-nutritive sweetener is almost a century old. Nature 1978, 271:493-495.

2. The straight facts on sweeteners. [http://www.thecoca-colacompany.com/ ourcompany/pdf/sweetener_fact_sheet.pdf].

3. Sugar substitutes. [http://www.pepsiproductfacts.com/sugarsub.php]

4. Lipson E: Chapter 1: Executive Summary. In Trends in the US market for sugar, sugar substitutes, and sweeteners Edited by: Packaged Facts 2008, $1-26$.

5. Freedonia: Freedonia focus on food and beverage additives The Freedonia Group I; 2010

6. Sievenpiper JL, Carleton AJ, Chatha S, Jiang HY, de Souza RJ, Beyene J, Kendall CW, Jenkins DJ: Heterogeneous effects of fructose on blood lipids in individuals with type 2 diabetes: systematic review and meta-analysis of experimental trials in humans. Diabetes Care 2009, 32:1930-1937.

7. Vermunt SH, Pasman WJ, Schaafsma G, Kardinaal AF: Effects of sugar intake on body weight: a review. Obes Rev 2003, 4:91-99.

8. Tuomilehto J, Lindstrom J, Eriksson JG, Valle $\Pi$, Hamalainen H, llanneParikka P, Keinanen-Kiukaanniemi S, Laakso M, Louheranta A, Rastas M, Salminen V, Uusitupa M, Finnish Diabetes Prevention Study Group: Prevention of type 2 diabetes mellitus by changes in lifestyle among subjects with impaired glucose tolerance. N Engl J Med 2001, 344:1343-1350.

9. Knowler WC, Barrett-Connor E, Fowler SE, Hamman RF, Lachin JM Walker EA, Nathan DM: Reduction in the incidence of type 2 diabetes with lifestyle intervention or metformin. N Engl J Med 2002, 346:393-403.

10. Gougeon R, Spidel M, Lee K, Field CJ: Canadian Diabetes Association National Nutrition Committee Technical Review: Non-nutritive intense sweeteners in diabetes management. Canadian Journal of Diabetes 2004, 28:385-399.

11. Moher D, Liberati A, Tetzlaff J, Altman DG: Preferred reporting items for systematic reviews and meta-analyses: the PRISMA statement. BMJ 2009, 339:b2535.

12. Jadad AR, Moore RA, Carrol D, Jenkinson C, Reynolds JM, Gavaghan DJ, McQuay HJ: Assessing the quality of reports of randomized clinical trials: Is blinding necessary? Control Clin Trials 1996, 17:1-12.

13. Schulze KF, Chalmers I, Hayes RJ, Altman DG: Empirical evidence of bias. JAMA 1995, 273:408-412.

14. Cho MK, Bero LA: The quality of drug studies published in symposium proceedings. Ann Intern Med 1996, 124:485-489.

15. Wiebe N, Vandermeer B, Platt RW, Klassen TP, Moher D, Barrowman NJ: A systematic review identifies a lack of standardization in methods for handling missing variance data. J Clin Epidemiol 2006, 59:342-353.

16. Egger M, Davey Smith G, Schneider M, Minder C: Bias in meta-analysis detected by a simple, graphical test. BMJ 1997, 315:629-634.

17. Renwick AG, Molinary SV: Sweet-taste receptors, low-energy sweeteners, glucose absorption and insulin release. Br J Nutr 2010, 104:1415-1420.

18. Borenstein M, Hedges LV, Higgins JPT, Rothstein HR: Chapter 16: Identifying and quantifying heterogeneity. In Introduction to MetaAnalysis. Volume 123. Wiley; 2009-124.

19. Lu G, Ades AE: Combination of direct and indirect evidence in mixed treatment comparisons. Stat Med 2004, 23:3105-3124.
20. Introduction to mixed treatment comparisons. [https://www.bris.ac.uk/ cobm/docs/intro\%20to\%20mtc.doc].

21. Dias S, Welton NJ, Caldwell DM, Ades AE: Checking consistency in mixed treatment comparison meta-analysis. Stat Med 2010, 29:932-944.

22. Prat-Larquemin L, Oppert JM, Bellisle F, Guy-Grand B: Sweet taste of aspartame and sucrose: effects on diet-induced thermogenesis. Appetite 2000, 34:245-251.

23. Melchior JC, Rigaud D, Colas-Linhart N, Petiet A, Girard A, Apfelbaum M: Immunoreactive beta-endorphin increases after an aspartame chocolate drink in healthy human subjects. PhysiolBehav 1991, 50:941-944.

24. Gonzalez-Ortiz M, Ramos-Zavala MG, Gonzalez-Lopez RC, RoblesCervantes JA, Martinez-Abundis E, Gonzalez-Ortiz M, Ramos-Zavala MG, Gonzalez-Lopez RC, Robles-Cervantes JA, Martinez-Abundis E: Effect of 2 liquid nutritional supplements for diabetes patients on postprandial glucose, insulin secretion, and insulin sensitivity in healthy individuals. JPEN J Parenter Enteral Nutr 2009, 33:67-70.

25. Horwitz DL, McLane M, Kobe P: Response to single dose of aspartame or saccharin by NIDDM patients. Diabetes Care 1988, 11:230-234.

26. Nguyen NU, Dumoulin G, Henriet MT, Berthelay S, Regnard J: Carbohydrate metabolism and urinary excretion of calcium and oxalate after ingestion of polyol sweeteners. J Clin Endocrinol Metab 1993, 77:388-392.

27. Hassinger W, Sauer G, Cordes U, Krause U, Beyer J, Baessler KH: The effects of equal caloric amounts of xylitol, sucrose and starch on insulin requirements and blood glucose levels in insulin-dependent diabetics. Diabetologia 1981, 21:37-40.

28. Rizkalla SW, Luo J, Wils D, Bruzzo F, Slama G: Glycaemic and insulinaemic responses to a new hydrogenated starch hydrolysate in healthy and type 2 diabetic subjects. Diabetes Metab 2002, 28(5):385-390.

29. Wheeler ML, Fineberg SE, Gibson R, Fineberg N: Metabolic response to oral challenge of hydrogenated starch hydrolysate versus glucose in diabetes.[see comment]. Diabetes Care 1990, 13:733-740.

30. Maki KC, Kanter M, Rains TM, Hess SP, Geohas J: Acute effects of low insulinemic sweeteners on postprandial insulin and glucose concentrations in obese men. Int J Food Sci Nutr 2009, 60(SUPPL 3):48-55.

31. Teff KL, Grudziak J, Townsend RR, Dunn TN, Grant RW, Adams SH, Keim NL, Cummings BP, Stanhope KL, Havel PJ: Endocrine and metabolic effects of consuming fructose- and glucose-sweetened beverages with meals in obese men and women: influence of insulin resistance on plasma triglyceride responses. J Clin Endocrinol Metab 2009, 94:1562-1569.

32. Bowen J, Noakes M, Clifton PM: Appetite hormones and energy intake in obese men after consumption of fructose, glucose and whey protein beverages. Int J Obes 2007, 31:1696-1703.

33. Chong MF, Fielding BA, Frayn KN, Chong MFF, Fielding BA, Frayn KN: Mechanisms for the acute effect of fructose on postprandial lipemia. Am J Clin Nutr 2007, 85:1511-1520.

34. Visvanathan R, Chen R, Garcia M, Horowitz M, Chapman I: The effects of drinks made from simple sugars on blood pressure in healthy older people.[see comment]. Br J Nutr 2005, 93:575-579.

35. Teff KL, Elliott SS, Tschop M, Kieffer TJ, Rader D, Heiman M, Townsend RR, Keim NL, D'Alessio D, Havel PJ: Dietary fructose reduces circulating insulin and leptin, attenuates postprandial suppression of ghrelin, and increases triglycerides in women. J Clin Endocrinol Metab 2004, 89:2963-2972.

36. Vozzo R, Baker B, Wittert GA, Wishart JM, Morris H, Horowitz M, Chapman I: Glycemic, hormone, and appetite responses to monosaccharide ingestion in patients with type 2 diabetes. Metabolism 2002, 51:949-957.

37. Spiller GA, Moynihan S, Butterfield G: Effects of sun-dried raisins on serum glucose: support for a convenient, plant-based snack food. Vegetarian Nutr 1998, 2:93-95.

38. Stewart SL, Black RM, Wolever TMS, Anderson GH: The relationship between glycaemic response to breakfast cereals and subjective appetite and food intake. Nutr Res 1997, 17:1249-1260.

39. Blaak EE, Saris WHM: Postprandial thermogenesis and substrate utilization after ingestion of different dietary carbohydrates. Metabolism 1996, 45:1235-1242.

40. Fukagawa NK, Veirs $H$, Langeloh G: Acute effects of fructose and glucose ingestion with and without caffeine in young and old humans. Metabolism 1995, 44:630-638.

41. Schwarz JM, Schutz $Y$, Piolino $V$, Schneider $H$, Felber JP, Jequier E: Thermogenesis in obese women: effect of fructose vs. glucose added to a meal. Am J Physiol 1992, 262:E394-E401. 
42. Bukar J, Mezitis NH, Saitas V, Pi-Sunyer FX: Frozen desserts and glycemic response in well-controlled NIDDM patients. Diabetes Care 1990, 13:382-385.

43. Georgakopoulos K, Katsilambros N, Fragaki M, Poulopoulou Z, Kimbouris J, Sfikakis $P$, Raptis S: Recovery from insulin-induced hypoglycemia after saccharose or glucose administration. Clin Physiol Biochem 1990, 8:267-272

44. Schwarz JM, Schutz Y, Froidevaux F, Acheson KJ, Jeanpretre N, Schneider $H$, Felber JP, Jequier $E$ : Thermogenesis in men and women induced by fructose vs glucose added to a meal. Am J Clin Nutr 1989, 49:667-674.

45. Simonson DC, Tappy L, Jequier E, Felber JP, DeFronzo RA: Normalization of carbohydrate-induced thermogenesis by fructose in insulin-resistant states. Am J Physiol 1988, 254:E201-E207.

46. Jansen RW, Penterman BJ, van Lier HJ, Hoefnagels WH: Blood pressure reduction after oral glucose loading and its relation to age, blood pressure and insulin. Am J Cardiol 1987, 60:1087-1091.

47. Tappy L, Randin JP, Felber JP, Chiolero R, Simonson DC, Jequier $E$, DeFronzo RA: Comparison of thermogenic effect of fructose and glucose in normal humans. Am J Physiol 1986, 250:E718-724

48. Samanta A, Burden AC, Jones GR: Plasma glucose responses to glucose, sucrose, and honey in patients with diabetes mellitus: an analysis of glycaemic and peak incremental indices. Diabet Med 1985, 2:371-373.

49. Bantle JP, Laine DC, Castle GW, Thomas JW, Hoogwerf BJ, Goetz FC Postprandial glucose and insulin responses to meals containing different carbohydrates in normal and diabetic subjects. N Engl J Med 1983, 309:7-12.

50. Crapo PA, Scarlett JA, Kolterman OG: Comparison of the metabolic responses to fructose and sucrose sweetened foods. Am J Clin Nutr 1982. 36:256-261.

51. Mann Jl, Truswell AS, Pimstone BL: The different effects of oral sucrose and glucose on alimentary lipaemia. Clin Sci 1971, 41:123-129.

52. Van Can JGP, lizerman TH, Van Loon LJC, Brouns F, Blaak EE: Reduced glycaemic and insulinaemic responses following isomaltulose ingestion: Implications for postprandial substrate use. Br J Nutr 2009, 102:1408-1413.

53. Qin LQ, Li J, Wang Y, Wang PY, Sato A, Kaneko T: Effect of repeated intake of disaccharides on glucose metabolism and insulin secretion in healthy adults - Comparison between sucrose and maltose. J Health Sci 2003, 49:226-228.

54. Stanhope KL, Griffen SC, Bair BR, Swarbrick MM, Keim NL, Havel PJ: Twentyfour-hour endocrine and metabolic profiles following consumption of high-fructose corn syrup-, sucrose-, fructose-, and glucose-sweetened beverages with meals. Am J Clin Nutr 2008, 87:1194-1203.

55. Melanson KJ, Zukley L, Lowndes J, Nguyen V, Angelopoulos TJ, Rippe JM: Effects of high-fructose corn syrup and sucrose consumption on circulating glucose, insulin, leptin, and ghrelin and on appetite in normal-weight women. Nutrition 2007, 23:103-112.

56. Kawai K, Yoshikawa H, Murayama Y, Okuda Y, Yamashita K: Usefulness of palatinose as a caloric sweetener for diabetic patients. Horm Metab Res 1989, 21:338-340.

57. Erkelens DW, Stofkooper A, Van DBE, Van D: Glycaemic effect of mono-, di- and polysaccharides in a mixed meal in diabetic patients. Neth J Med 1985, 28:157-163.

58. Grysman A, Carlson T, Wolever TM, Wolever TMS: Effects of sucromalt on postprandial responses in human subjects. Eur J Clin Nutr 2008, 62:1364-1371.

59. Munstedt K, Sheybani B, Hauenschild A, Bruggmann D, Bretzel RG, Winter D: Effects of basswood honey, honey-comparable glucosefructose solution, and oral glucose tolerance test solution on serum insulin, glucose, and C-peptide concentrations in healthy subjects. J Med Food 2008, 11:424-428.

60. Bowker SL, Majumdar SR, Johnson JA: Systematic review of indicators and measurements used in controlled studies of quality improvement for type 2 diabetes. Can J Diabetes 2005, 29:230-238.

61. Reid M, Hammersley R, Hill AJ, Skidmore P: Long-term dietary compensation for added sugar: effects of supplementary sucrose drinks over a 4-week period. Br J Nutr 2007, 97:193-203.

62. Chantelau EA, Gosseringer G, Sonnenberg GE, Berger M: Moderate intake of sucrose does not impair metabolic control in pump-treated diabetic out-patients. Diabetologia 1985, 28:204-207.

63. Raben A, Vasilaras TH, Moller AC, Astrup A: Sucrose compared with artificial sweeteners: different effects on ad libitum food intake and body weight after 10 wk of supplementation in overweight subjects. Am J Clin Nutr 2002, 76:721-729.

64. Ngo Sock ET, Le KA, Ith M, Kreis R, Boesch C, Tappy L: Effects of a shortterm overfeeding with fructose or glucose in healthy young males. $\mathrm{Br} J$ Nutr 2010, 103:939-943.

65. Bantle JP, Raatz SK, Thomas W, Georgopoulos A: Effects of dietary fructose on plasma lipids in healthy subjects. Am J Clin Nutr 2000, 72:1128-1134.

66. Macdonald I, Taylor J: Differences in body weight loss on diets containing either sucrose or glucose syrup. Guys Hosp Rep 1973, 122:155-159.

67. Bantle JP, Laine DC, Thomas JW: Metabolic effects of dietary fructose and sucrose in types I and II diabetic subjects. JAMA 1986, 256:3241-3246.

68. Okuno M, Kim MK, Mizu M, Mori M, Mori H, Yamori Y: Palatinose-blended sugar compared with sucrose: different effects on insulin sensitivity after 12 weeks supplementation in sedentary adults. Int J Food Sci Nutr 2010, 61:643-651.

69. Yaghoobi N, Al-Waili N, Ghayour-Mobarhan M, Parizadeh SM, Abasalti Z, Yaghoobi Z, Yaghoobi F, Esmaeili H, Kazemi-Bajestani SM, Aghasizadeh R, Saloom KY, Ferns GA: Natural honey and cardiovascular risk factors; effects on blood glucose, cholesterol, triacylglycerole, CRP, and body weight compared with sucrose. ScientificWorldJournal 2008, 8:463-469.

70. Boesch C, Ith M, Jung B, Bruegger K, Erban S, Diamantis I, Kreis R, Bar A: Effect of oral D-tagatose on liver volume and hepatic glycogen accumulation in healthy male volunteers. Regul Toxicol Pharmacol 2001, 33:257-267.

71. Luo J, Van Yperselle M, Rizkalla SW, Rossi F, Bornet FR, Slama G: Chronic consumption of short-chain fructooligosaccharides does not affect basal hepatic glucose production or insulin resistance in type 2 diabetics. $J$ Nutr 2000, 130:1572-1577.

72. Luo J, Rizkalla SW, Alamowitch C, Boussairi A, Blayo A, Barry JL, Laffitte A, Guyon F, Bornet FR, Slama G: Chronic consumption of short-chain fructooligosaccharides by healthy subjects decreased basal hepatic glucose production but had no effect on insulin-stimulated glucose metabolism. Am j clin nutr 1996, 63:939-945.

73. Alles MS, de Roos NM, Bakx JC, van de Lisdonk E, Zock PL, Hautvast GA: Consumption of fructooligosaccharides does not favorably affect blood glucose and serum lipid concentrations in patients with type 2 diabetes. Am J Clin Nutr 1999, 69:64-69.

74. Pearson ES: The application of statistical methods to industrial standardization and quality controls. London, UK: B.S. 600, British Standards Institution; 1960.

75. Bellisle F, Drewnowski A: Intense sweeteners, energy intake and the control of body weight. Eur J Clin Nutr 2007, 61:691-700.

76. Smeets PA, de Graaf C, Stafleu A, van Osch MJ, van der Grond J: Functional magnetic resonance imaging of human hypothalamic responses to sweet taste and calories. Am J Clin Nutr 2005, 82:1011-1016.

77. Tam J, Fukumura D, Jain RK: A mathematical model of murine metabolic regulation by leptin: energy balance and defense of a stable body weight. Cell Metab 2009, 9:52-63.

78. Sullivan EL, Cameron JL: A rapidly occurring compensatory decrease in physical activity counteracts diet-induced weight loss in female monkeys. Am J Physiol Regul Integr Comp Physiol 2010, 298:R1068-1074.

79. Erlanson-Albertsson C: How palatable food disrupts appetite regulation. Basic Clin Pharamcol Toxicol 2005, 97:61-73.

80. Black RM, Leiter LA, Anderson GH: Consuming aspartame with and without taste: differential effects on appetite and food intake of young adult males. Physiol Behav 1993, 53:459-466.

81. Buchwald $H$, Avidor $Y$, Braunwald $E$, Jensen MD, Pories W, Fahrbach $K$, Schoelles K: Bariatric surgery: a systematic review and meta-analysis. JAMA 2004, 292:1724-1737.

82. Guh DP, Zhang W, Bansback N, Amarsi Z, Birmingham CL, Anis AH: The incidence of co-morbidities related to obesity and overweight: a systematic review and meta-analysis. BMC Public Health 2009, 9:88.

83. Gougeon R, Spidel M, Lee K, Field CJ: Canadian Diabetes Association National Nutrition Committee Technical Review: Non-nutritive Intense Sweeteners in Diabetes Management. Can J Diabetes 2004, 28:385-399.

84. Wolever T, Barbeau M, Charron S, Harrigan K, Leung S, Madrick B, Taillefer T, Seto C: Guidelines for the nutritional management of diabetes mellitus in the new millennium: A position statement by the Canadian Diabetes Association. Can J Diabetes Care 1999, 23:56-69. 
85. Calorie Control Council. [http://www.caloriecontrol.org/sweeteners-andlite].

86. Powers MA, Crapo PA: The fructose story. Diabetes Educ 1982, 7:22-25.

87. Lycasin maltitol syrups. [http://www.roquette-food.com/delia-CMS/ search_product/topic_id-1720/article_id-3653/product_id-1208/.

88. Glucerna 1.2 Cal Monograph. [http://abbottnutrition.com/Downloads/Gluc1_2-Cal.pdf].

89. Sweeteners. [http://www.elmhurst.edu/ chm/vchembook/549sweet.html].

90. Carbohydrates and the sweeteners of honey. [http://www.honey.com/ nhb/technical/technical-reference/].

91. PALATINOSETM -The functional carbohydrate providing better energy. [http://www.beneo-palatinit.com/en/Food_Ingredients/Isomaltulose/ What_is_Isomaltulose/Palatinose-Brochure_EN_1.pdf].

92. Trehalose. [http://www.caloriecontrol.org/sweeteners-and-lite/sugarsubstitutes/other-sweeteners/trehalose].

\section{Pre-publication history}

The pre-publication history for this paper can be accessed here: http://www.biomedcentral.com/1741-7015/9/123/prepub

doi:10.1186/1741-7015-9-123

Cite this article as: Wiebe et al:: A systematic review on the effect of sweeteners on glycemic response and clinically relevant outcomes. BMC Medicine 2011 9:123.

\section{Submit your next manuscript to BioMed Central} and take full advantage of:

- Convenient online submission

- Thorough peer review

- No space constraints or color figure charges

- Immediate publication on acceptance

- Inclusion in PubMed, CAS, Scopus and Google Scholar

- Research which is freely available for redistribution

Submit your manuscript at www.biomedcentral.com/submit 